Ultrafast photoelectron migration in dye-sensitized solar cells: Influence of the binding mode and many-body interactions

G. Hermann and J. C. Tremblay

Citation: J. Chem. Phys. 145, 174704 (2016); doi: 10.1063/1.4966260

View online: http://dx.doi.org/10.1063/1.4966260

View Table of Contents: http://aip.scitation.org/toc/jcp/145/17

Published by the American Institute of Physics

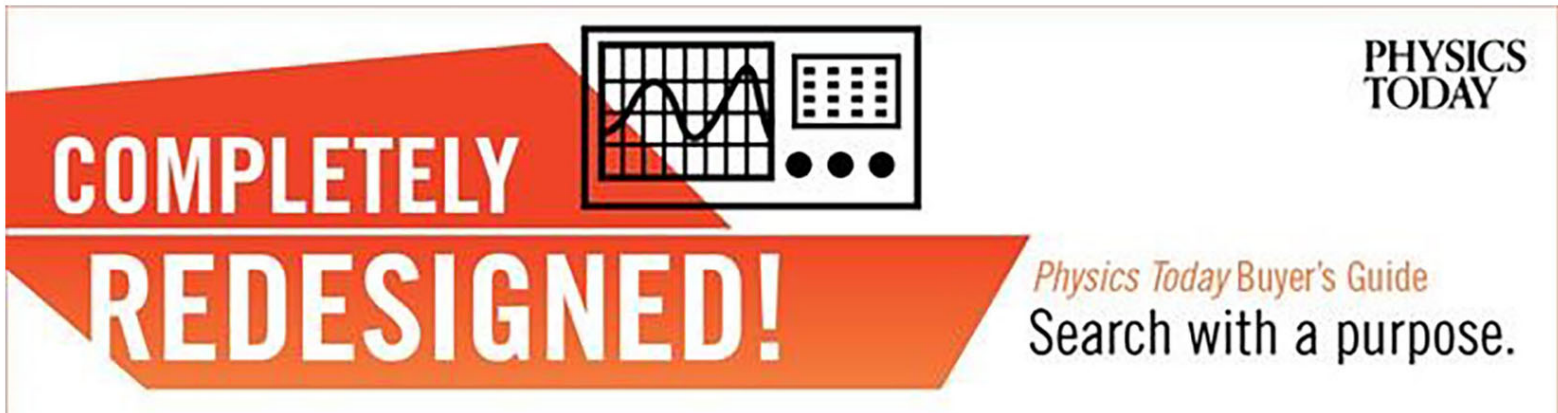




\title{
Ultrafast photoelectron migration in dye-sensitized solar cells: Influence of the binding mode and many-body interactions
}

\author{
G. Hermann ${ }^{\text {a) }}$ and J. C. Tremblay ${ }^{\text {b) }}$ \\ Institut für Chemie und Biochemie, Freie Universität Berlin, Takustraße 3, 14195 Berlin, Germany
}

(Received 29 June 2016; accepted 7 October 2016; published online 3 November 2016)

\begin{abstract}
In the present contribution, the ultrafast photoinduced electron migration dynamics at the interface between an alizarin dye and an anatase $\mathrm{TiO}_{2}$ thin film is investigated from first principles. Comparison between a time-dependent many-electron configuration interaction ansatz and a single active electron approach sheds light on the importance of many-body effects, stemming from uniquely defined initial conditions prior to photoexcitation. Particular emphasis is put on understanding the influence of the binding mode on the migration process. The dynamics is analyzed on the basis of a recently introduced toolset in the form of electron yields, electronic fluxes, and flux densities, to reveal microscopic details of the electron migration mechanism. From the many-body perspective, insight into the nature of electron-electron and hole-hole interactions during the charge transfer process is obtained. The present results reveal that the single active electron approach yields quantitatively and phenomenologically similar results as the many-electron ansatz. Furthermore, the charge migration processes in the dye- $\mathrm{TiO}_{2}$ model clusters with different binding modes exhibit similar mechanistic pathways but on largely different time scales. Published by AIP Publishing. [http://dx.doi.org/10.1063/1.4966260]
\end{abstract}

\section{INTRODUCTION}

Photoinduced heterogeneous electron transfer between molecular adsorbates and semiconductor materials is an important fundamental process, relevant to a variety of practical applications in many fields. ${ }^{1,2}$ Significant research effort has been devoted to the characterization and understanding of the electron transfer processes in dyesensitized solar cells (DSSCs) due to its importance for the solar energy conversion..$^{3-9}$ In general, DSSCs mainly consist of transition-metal or organic dye molecules that are adsorbed to a mesoporous nanocrystalline thin film or nanoparticle of a wide bandgap semiconductor, typically titanium dioxide $\left(\mathrm{TiO}_{2}\right)$. In these Grätzel-type solar cells, the electron transfer is initiated by a localized optical excitation on the chromophore to a state hybridized with the semiconductor conduction band. The photoelectron is then transferred from the donor orbital to the support material, ultimately leading to an irreversible electron injection. A series of experimental studies has given evidence that this electron injection at the dyesemiconductor interface often proceeds on the femtosecond time scale. ${ }^{6,10-14}$

In recent years, several theoretical methods have been proposed to characterize the electron injection process in real time, which were applied to a number of different DSSC systems. ${ }^{15-19}$ A complete microscopic understanding of this ultrafast dynamical process necessitates a detailed description of the electronic structure of the dye-substrate systems and time-resolved simulations of the injection process. ${ }^{20}$ Commonly, two strategies are pursued: the fully

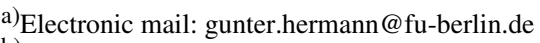

${ }^{b)}$ Electronic mail: jctremblay@zedat.fu-berlin.de
}

quantum-mechanical description of the coupled electronnuclear dynamics based on model Hamiltonians, or the firstprinciple simulation of electron dynamics combined with a classical treatment of nuclear motions. Both have been employed to investigate various aspects affecting the operation and efficiency of DSSCs, including charge recombination, ${ }^{21}$ thermal fluctuations, ${ }^{22}$ the influence of the anchor group, ${ }^{23}$ and the effect of the reorganization energy. ${ }^{24}$ Recently, we introduced a density based toolkit to analyze and visualize the flow of electrons during charge migration processes, which allowed unraveling the microscopic mechanism of electronic injection in DSSCs. ${ }^{25}$

A potentially insightful complement to these theoretical investigations is the explicit examination of many-body effects on the electron dynamics during the transfer process. Towards this end, we provide here a comparison between a single active electron approach and a wave function ansatz to simulate the electron injection in real time, within the clamped nuclei approximation. For the latter, a variant of the explicitly time-dependent configuration interaction method is used, which was shown to be an accurate tool to model light-induced dynamics of charge migration processes at an atomistic level of detail. ${ }^{26-30}$ Retaining a single active electron, the many-body wave function ansatz reduces to a set of uncorrelated single-particle equations of motion. These are reminiscent of the time-dependent Kohn-Sham (KS) formulation used by others to investigate electron transfer in more complex DSSC model systems. ${ }^{17,31-33}$ Whereas the influence of vibrational fluctuations on the electron dynamics in DSSCs is well documented, ${ }^{20,34,35}$ the main characteristics of the injection process can be captured within a purely electronic picture for weakly coupled systems. ${ }^{34,35}$ The manybody effects are expected to influence the dynamics in two 
ways: (i) via electronic coherences and interference effects in the equations of motion and (ii) in the reconstruction of the time-dependent electron density. The first effect depends on the specific energetic profiles associated with the uncorrelated vs. correlated wave functions and influences the time scales of the injection process. The latter can be revealed by analyzing the electron flow during the injection process. Here, we propose a new procedure to separate the various contributions of the many-body wave function, providing a meaningful comparison between the one- and many-electron approaches and revealing dynamical electron and hole correlations.

A representative model for investigating electron transfer processes is the alizarin- $\mathrm{TiO}_{2}$ complex, which has attracted huge interest over the years. Besides numerous experimental and computational studies on its optical properties, i.e., electronic absorption spectra, ${ }^{11,25,34,36-39}$ the heterogeneous electron transfer process has been investigated in great detail. ${ }^{13,35,40-44}$ An electron injection time of 60 fs was reported by Wachtveitl and co-workers for alizarin anchored to a $\mathrm{TiO}_{2}$ thin film. ${ }^{42}$ Real-time dynamical simulations of the interfacial electron transfer in alizarin$\mathrm{TiO}_{2}$ are typically described by coupled electron-nuclear equations of motion..$^{34,35,43,45}$ While these treatments have provided valuable information about several dynamical features, conclusions on the impact of many-body effects on the electron structure and the dynamical mechanism during the charge migration cannot be drawn. In previous theoretical work, ${ }^{20}$ the bonding motif and orientation have been shown to have a great impact on the electronic structure at interface. In particular, catechol derivatives (e.g., alizarin or coumarin) were found to form a strong chemical bond with the semiconductor $\left(\mathrm{TiO}_{2}\right)$, with some degree of charge transfer from the anchoring $\mathrm{Ti}$ atoms to the dye. Both monodentate and bidentate bonding motifs proved similarly stable when allowing for relaxation of the adsorption site. While the optical spectrum exhibits a similar red-shift upon adsorption for both anchoring types, the energetic alignment and the spatial distribution of acceptor and donor states depend more strongly on the bonding motif. These are pivotal properties influencing the electron injection process. To the best of our knowledge, no information about the flow of electrons during this process is available. Consequently, the influence of the bonding motifs can only be inferred qualitatively. In the following, we will provide microscopic insight into how different bonding motifs affect the charge migration process after laser excitations from well-defined initial conditions.

The remainder of this paper is organized as follows. Sec. II briefly introduces the theoretical outline, including the electronic structure methods to characterize the model systems, the time-dependent single active electron approach, and the time-dependent many-body configuration interaction method (TDCI), as well as the analysis tools to investigate the charge migration process. In the subsequent paragraphs, the results of the static calculations and the laser-driven electron dynamics are presented and analyzed, followed by a conclusion summarizing the most important findings.

\section{THEORY}

\section{A. Model system}

Our efforts focus on the dye-semiconductor complex alizarin- $\mathrm{TiO}_{2}$ as a representative model for dye sensitized solar cells due to its promising electronic properties. The interaction between alizarin and a thin film of titania is modeled using a finite cluster carved out of the bulk anatase ${ }^{46}$ Following the work of Li et al. ${ }^{35}$ we choose a cluster of ten $\mathrm{TiO}_{2}$ units for an optimal balance between minimal boundary effects and low computational costs. To ensure satisfactory coordination of all cluster atoms, all dangling bonds at the boundary oxygen or titanium atoms are saturated with hydrogen atoms or hydroxyl groups, respectively. Anchoring of the alizarin molecule $\mathrm{C}_{14} \mathrm{H}_{8} \mathrm{O}_{4}$ onto the terrace site of the substrate $\left(\mathrm{TiO}_{2}\right)_{10}\left(\mathrm{H}_{2} \mathrm{O}\right)_{20}$, i.e., the (101) surface of anatase, is achieved through its hydroxyl groups accompanied by loss of water (dissociative adsorption). ${ }^{20,47}$ In order to determine the effect of the binding mode on the direct injection process, we construct several stoichiometric clusters with different dissociative anchoring motifs. Their influence towards the electronic properties of the system and the transient dynamics is a key aspect of the present investigations.

Structure optimization of the neutral dye-semiconductor complexes is performed at the density functional theory (DFT) level with the B3LYP functional ${ }^{48}$ and the def2SVP basis set. ${ }^{49,50}$ During this procedure, the dye and the anchoring titanium atoms as well as the boundary hydrogen atoms could freely relax, while all other degrees of freedom are kept frozen. To verify the suitability of the model system, electronic absorption spectra are computed using linear response time-dependent density functional theory (TDDFT) ${ }^{51}$ The particular choice of TDDFT/B3LYP has been previously applied to accurately predict the electronic and spectroscopic properties in dye-semiconductor systems, ${ }^{38,52-56}$ and in particular also for alizarin- $\mathrm{TiO}_{2}$ complexes. ${ }^{25,39,43}$ Besides its favorable cost-to-accuracy ratio, this approach includes electron correlation effects, a fact that will be exploited in the theoretical treatment described in Sec. II B. All calculations are carried out with the TURBOMOLE package, ${ }^{57}$ and post-processing is performed using our open source Python toolbox ORBKIT, ${ }^{58}$ to be described below.

\section{B. Electron dynamics}

In this work, we perform real-time, fully atomistic simulations of a laser-induced electron migration process in a model DSSC for different bonding motifs. Emphasis is put on the comparison between two methods: a timedependent many-electron configuration interaction algorithm and a time-dependent single active electron approach. The aim is to reveal the importance of electronic correlation on the direct injection process. To simplify comparison between both methods, the nuclear configurations are kept fixed for the model clusters throughout the study. The timeevolution of laser-driven electronic wave packets is treated by solving the time-dependent, non-relativistic Schrödinger equation, ${ }^{59}$ 


$$
l \frac{\partial}{\partial t} \Psi_{\mathrm{el}}\left(\mathbf{r}^{N}, t\right)=\hat{H}\left(\mathbf{r}^{N}, t\right) \Psi_{\mathrm{el}}\left(\mathbf{r}^{N}, t\right),
$$

with $\Psi_{\mathrm{el}}\left(\mathbf{r}^{N}, t\right)$ as the total electronic wave function of the $N$-electron system. $\hat{H}\left(\mathbf{r}^{N}, t\right)$ is the time-dependent Hamiltonian, which includes the interaction with a timedependent electric field $\mathbf{F}(t)$ within the semi-classical dipole approximation. Boundary conditions to simulate electron loss at the bottom edge of the cluster are enforced by adding a complex absorbing potential $\hat{W}_{\mathrm{CAP}}$ to the Hamiltonian as

$$
\hat{H}\left(\mathbf{r}^{N}, t\right)=\hat{H}_{\mathrm{el}}\left(\mathbf{r}^{N}\right)-\hat{\mu} \mathbf{F}(t)-\hat{W}_{\mathrm{CAP}} .
$$

Here, $\hat{H}_{\mathrm{el}}\left(\mathbf{r}^{N}\right)$ is the field-free electronic Hamiltonian, and $\hat{\boldsymbol{\mu}}$ refers to the molecular dipole operator. The applied electric field $\mathbf{F}(t)$ is expressed as a linearly polarized laser pulse along the main molecular axis (here the $x$ axis) with a sine square envelope,

$$
\begin{aligned}
\mathbf{F}(t) & =\left(\begin{array}{c}
f_{x}(t) \\
0 \\
0
\end{array}\right) \cos (\omega t), \\
f_{x}(t) & = \begin{cases}f_{0 x} \sin ^{2}\left(\frac{\pi t}{t_{f}}\right) & \text { if } 0<t<t_{f}, \\
0 & \text { else, }\end{cases}
\end{aligned}
$$

where $\omega$ is the carrier frequency of the laser field, $t_{f}$ denotes the pulse length, and $f_{0 x}$ designates the field amplitude.

To describe the electronic structure out of equilibrium, the time-dependent electronic wave function $\Psi_{\mathrm{el}}\left(\mathbf{r}^{N}, t\right)$ is expanded in general terms as follows:

$$
\Psi_{\mathrm{el}}\left(\mathbf{r}^{N}, t\right)=\sum_{\lambda=0} B_{\lambda}(t) \Phi_{\lambda}\left(\mathbf{r}^{N}\right),
$$

with $B_{\lambda}(t)$ as time-dependent expansion coefficients and $\Phi_{\lambda}\left(\mathbf{r}^{N}\right)$ as the time-independent wave function of state $\lambda$ that satisfies the stationary electronic Schrödinger equation,

$$
\hat{H}_{\mathrm{el}}\left(\mathbf{r}^{N}\right) \Phi_{\lambda}\left(\mathbf{r}^{N}\right)=E_{\lambda} \Phi_{\lambda}\left(\mathbf{r}^{N}\right) .
$$

The propagation of the coefficients $B_{\lambda}(t)$ is determined by direct numerical integration of Eq. (1) using an adaptive Runge-Kutta algorithm in the interaction picture. Implementation details are described elsewhere. ${ }^{28,60,61}$

In the many-body TDCI ansatz, each time-independent electron wave function $\Phi_{\lambda}\left(\mathbf{r}^{N}\right)$ takes the form of an $\mathrm{N}$-electron eigenstate at the configuration interaction singles (CIS) level. ${ }^{62}$ That is, a given excited state of the electronic Hamiltonian is written as a linear combination of the ground state Slater determinant $\phi_{0}$ and singly excited configuration state functions $\phi_{a}^{r}$,

$\Phi_{\lambda}^{\mathrm{CIS}}\left(\mathbf{r}^{N}\right)=D_{0}^{(\lambda)} \phi_{0}\left(\mathbf{r}^{N}\right)+\sum_{a=L}^{N / 2} \sum_{r=N / 2+1}^{M} D_{a}^{r(\lambda)} \phi_{a}^{r}\left(\mathbf{r}^{N}\right)$,

where $\left\{D_{0}^{(\lambda)}, D_{a}^{r(\lambda)}\right\}$ are the expansion coefficients, $N$ is the number of electrons, and $L$ and $M$ denote the lowest occupied and highest unoccupied orbitals, respectively. The singly excited configuration state functions $\phi_{a}^{r}\left(\mathbf{r}^{N}\right)$ are derived from the reference configuration $\phi_{0}\left(\mathbf{r}^{N}\right)$ by moving an electron from an occupied MO $a$ to an unoccupied MO $r$. As was shown in recent work, ${ }^{63,64}$ the expansion coefficients can be alternatively obtained from linear response TDDFT. This allows for more flexibility in the definition of the electronic Hamiltonian, Eq. (5), which can provide a better description of electron correlation in the reference, $\phi_{0}\left(\mathbf{r}^{N}\right)=\Psi_{0}^{\mathrm{DFT}}\left(\mathbf{r}^{N}\right)$, thus providing better energetics for the excited states. The energies obtained from linear response TDDFT are good approximations to the eigenvalues of the $N$-electron Hamiltonian, Eq. (5). The associated response coefficients are renormalized to yield an approximate CIS wave function of the form of Eq. (6) on the basis of Kohn-Sham orbitals. Although states with double excitation character can be potentially accessed by excitation using an electric field (see, e.g., Ref. 65 and references therein), linear response TDDFT was shown to provide satisfactory agreement with benchmark equation of motion coupled-cluster singles doubles calculations in typical chromophores used in DSSCs. ${ }^{66}$

To the benefit of a computationally inexpensive alternative to this many-body ansatz, we investigate the properties of a time-dependent single active electron approach. Here, the time-independent electron wave function $\Phi_{\lambda}\left(\mathbf{r}^{N}\right)$ is represented by a one-electron Kohn-Sham (KS) orbital $\varphi_{n}(\mathbf{r})$. Hence, it is referred to as TDKS approach. Both the TDKS and TDCI wave functions are built from the same time-independent KS orbitals and the same excited configurations. They are used as a static basis to represent the electronic wave packet, Eq. (4). The TDCI wave function is a linear combination of static CIS eigenstates, which are linear combinations of configuration state functions, built on the static KS orbitals. Here, all possible single electronhole excitations from occupied to virtual (KS) orbitals are considered. The time-dependence comes only from the expansion coefficients in this otherwise static basis. The TDKS wave function is strictly equivalent to a TDCI wave function for which only a single occupied orbital can be excited to a restricted set of virtual orbitals. The TDKS wave function thus spans a subset of the configurations used in the TDCI ansatz, in which all electrons but one are frozen. This is particularly convenient since it ensures that the TDKS and TDCI simulations have exactly the same initial conditions: the ground state Slater determinant obtained from a single KohnSham density functional theory calculation. The electronic Hamiltonian in the TDKS ansatz neglects correlations between the electron/hole pair excitations. These are otherwise present in the TDCI Hamiltonian, since all excited configurations are taken into account.

According to the MO-LCAO (Molecular Orbital-Linear Combination of Atomic Orbitals) ansatz, ${ }^{67}$ the KS orbitals can be written as a linear combination of a finite set of atomic orbitals $\psi_{i}\left(\mathbf{r}-\mathbf{R}_{A}\right)$,

$$
\Phi_{\lambda}^{\mathrm{KS}}(\mathbf{r}) \cong \varphi_{n}(\mathbf{r})=\sum_{A}^{N_{\mathrm{A}}} \sum_{i}^{N_{\mathrm{AO}}} C_{n i} \psi_{i}\left(\mathbf{r}-\mathbf{R}_{A}\right),
$$

where $C_{n i}$ denotes the $i$ th $\mathrm{MO}$ coefficient for the MO $n, N_{\mathrm{A}}$ stands for the number of atoms, and $N_{\mathrm{AO}}$ is the number of atomic orbitals. In the TDKS approach, the time-dependent one-electron wave function $\Psi_{\mathrm{el}}^{\mathrm{TDKS}}(\mathbf{r}, t)$ fulfills a one-electron variant of Eq. (1), and the KS orbital energies are used as state 
energies. Within the single active electron approximation, the photoinduced electron dynamics is described as a pure one-electron process. Hence, the initial condition comprises a single occupied active orbital, usually the HOMO, while the excitation dynamics in Eq. (4) requires appropriate selection of virtual KS orbitals $\varphi_{n}(\mathbf{r})$. The suitability of this approach for the proper characterization of photoinduced electron transfer processes can be substantiated by the fact that the photoexcitation in DSSCs is usually dominated by an intramolecular transition between two localized orbitals at the dye. This was confirmed by linear-response TDDFT calculations in previous investigations of alizarin- $\mathrm{TiO}_{2}$ solar cells. $^{25}$

In order to simulate the electron transfer process at an atomistic level, a finite dye-semiconductor model cluster is employed. To prevent artificial reflection at the cluster edges, absorbing boundary conditions are enforced using a complex absorbing potential on the hydrogen atoms $(\mathrm{H})$ at the $\mathrm{TiO}_{2}$ cluster edges. This allows to mimic the irreversible migration of an electron from the dye-cluster complex to an extended $\mathrm{TiO}_{2}$ thin film, where the electron preferably diffuses into the infinite substrate. The absorbing potential is defined using a spatial projector formalism ${ }^{25,35}$

$$
\hat{W}_{\mathrm{CAP}}=\gamma_{\mathrm{CAP}} \hat{P}_{\text {sink }},
$$

where $\gamma_{\mathrm{CAP}}$ is a user-defined parameter. The spatial projector $\hat{P}_{\text {sink }}$ builds on the definition of the molecular orbitals as a linear combination of atomic orbitals, Eq. (7). The projector solely affects the hydrogen atoms at the edges of the cluster to simulate the contact of the cluster with an infinite substrate. The associated relaxation rates in the TDKS approach can be formulated as

$$
\begin{aligned}
\Gamma_{n}^{\mathrm{TDKS}} & =\gamma_{\mathrm{CAP}}\left\langle\varphi_{n}\left|\hat{P}_{\text {sink }}\right| \varphi_{n}\right\rangle \\
& =\gamma_{\mathrm{CAP}} \sum_{A \in \mathrm{H}}^{N_{\mathrm{A}}} \sum_{i}^{N_{\mathrm{AO}}} \sum_{j_{A}}^{N_{\mathrm{AO}}} C_{n j_{A}} C_{n i} S_{j_{A} i},
\end{aligned}
$$

where $S_{j_{A} i}=\left\langle\psi_{j_{A}} \mid \psi_{i}\right\rangle$ designates an element of the atomic orbital overlap matrix. Since the absorption through the absorbing potential is an incoherent mechanism, the respective relaxation rates within the TDCI method are simply the weighted sum of their orbital contributions

$$
\Gamma_{\lambda}^{\mathrm{TDCI}}=\sum_{a=L}^{N / 2} \sum_{r=N / 2+1}^{M}\left(D_{a}^{r(\lambda)}\right)^{2} \Gamma_{r}^{\mathrm{TDKS}} .
$$

For the photoinduced electron transfer dynamics, $\gamma_{\mathrm{CAP}}$ is adjusted so that the relaxation rate of the reference excited state matches the experimentally observed injection time for alizarin attached to a $\mathrm{TiO}_{2}$ thin film $(60 \mathrm{fs}) .{ }^{42}$ This has the effect of damping all dynamical processes slower than this cutoff time. The ultrafast coherent processes remain otherwise unaffected during the dynamics.

\section{Analysis toolkit}

The conceptual understanding of the ultrafast heterogeneous electron migration process in DSSCs is the cornerstone in the development of novel solar cells and thus in the improvement of their photon-to-current conversion efficiency. A complete picture of the electron transfer mechanism can be only obtained through the analysis of time-resolved simulations. For that purpose, we recently developed a set of tools (e.g., electronic yields and electronic flux densities) to help visualize the underlying dynamical mechanism. The prevailing quantity for the visualization of electronic motions in molecular systems is the time-dependent one-electron density, which takes the following form: ${ }^{68}$

$$
\rho(\mathbf{r}, t)=\int \Psi_{\mathrm{el}}\left(\mathbf{r}^{N}, t\right) \Psi_{\mathrm{el}}^{\dagger}\left(\mathbf{r}^{N}, t\right) \mathrm{d} \mathbf{r}^{N-1} .
$$

To shed light on the heterogeneous character of the interfacial charge migration, the time-dependent electron density can be spatially decomposed using the Voronoi partitioning scheme. ${ }^{69}$ This formalism divides the space into regions assigned to a certain atom or fragment. These particular domains of space are referred to as Voronoi polyhedrons and are defined as the set of points in space closest to the selected atom or fragment $V$. The spatial integration of the electron density over these Voronoi cells leads to the number of electrons $N_{V}$ in the respective volume at any given time,

$$
N_{V}(t)=\int_{\substack{\text { Voronoi } \\ \text { cell of } V}} \mathrm{~d} \mathbf{r} \rho(\mathbf{r}, t) .
$$

Another central quantity for the visual representation of real-time dynamics of electronic motion induced by a time-dependent perturbation is the electronic flux density $\mathbf{j}(\mathbf{r}, t)$. This vector field illustrates the spatial flow of the electron density, revealing information about its magnitude and direction at each point in space. It is related to the velocity field via the one-electron density, $\mathbf{j}(\mathbf{r}, t)=\rho(\mathbf{r}, t) \mathbf{v}(\mathbf{r}, t)$. In zero gauge, the electronic flux density $\mathbf{j}(\mathbf{r}, t)$ is defined as $^{68,70,71}$

$\mathbf{j}(\mathbf{r}, t)=-\frac{\hbar}{m_{e}} \operatorname{Im}\left[\int \Psi_{\mathrm{el}}\left(\mathbf{r}^{N}, t\right) \vec{\nabla} \Psi_{\mathrm{el}}^{\dagger}\left(\mathbf{r}^{N}, t\right) \mathrm{d} \mathbf{r}^{N-1}\right]$.

From the time-dependent many-body wave function, Eqs. (4) and (6), the flux density can be computed as

$$
\begin{aligned}
\mathbf{j}^{\mathrm{TDCI}}(\mathbf{r}, t)= & -\frac{\hbar}{m_{e}} \sum_{\lambda \neq v} \operatorname{Im}\left[B_{\lambda}(t) B_{v}^{\dagger}(t)\right] \\
& \times \int\left(\Phi_{\lambda}^{\mathrm{TDCI}}\left(\mathbf{r}^{N}\right) \vec{\nabla} \Phi_{v}^{\mathrm{TDCI}}\left(\mathbf{r}^{N}\right)\right) \mathrm{d} \mathbf{r}^{N-1} \\
= & -\frac{2 \hbar}{m_{e}} \sum_{\lambda<v} \operatorname{Im}\left[B_{\lambda}(t) B_{v}^{\dagger}(t)\right] \\
& \times \int\left(\Phi_{\lambda}^{\mathrm{TDCI}}\left(\mathbf{r}^{N}\right) \vec{\nabla} \Phi_{v}^{\mathrm{TDCI}}\left(\mathbf{r}^{N}\right)\right) \mathrm{d} \mathbf{r}^{N-1} .
\end{aligned}
$$

The second line follows from the anti-symmetry of the gradient operator. Inserting instead the time-dependent electronic wave function definition of the one-electron TDKS approach, Eqs. (4) and (7), the approximate expression for the flux density is alternatively given by

$$
\mathbf{j}^{\mathrm{TDKS}}(\mathbf{r}, t)=-\frac{2 \hbar}{m_{e}} \sum_{\lambda<v} \operatorname{Im}\left[B_{\lambda}(t) B_{v}(t)\right]\left(\varphi_{\lambda}(\mathbf{r}) \vec{\nabla} \varphi_{v}(\mathbf{r})\right) .
$$


Note that in both expressions, Eqs. (14) and (15), the time evolution of the electronic flux density is governed by the time-dependence of the coefficients $B_{\lambda}(t)$.

Regarding Eq. (14), the construction of the determinantal functions in the CIS wave function $\Phi_{\lambda}^{\mathrm{CIS}}\left(\mathbf{r}^{N}\right)$ (cf. Eq. (6)) allows a detailed analysis of $\mathbf{j}^{\mathrm{TDCI}}(\mathbf{r}, t)$. For this purpose, Eq. (14) is decomposed into four contributions

$$
\begin{aligned}
\int \Phi_{\lambda}^{\mathrm{CIS}} & \left(\mathbf{r}^{N}\right) \vec{\nabla} \Phi_{\nu}^{\mathrm{CIS}}\left(\mathbf{r}^{N}\right) \mathrm{d} \mathbf{r}^{N-1} \\
= & \sum_{a r} D_{0}^{(\lambda)} D_{a}^{r(v)}\left\langle\phi_{0}|\vec{\nabla}| \phi_{a}^{r}\right\rangle_{\mathbf{r}} \\
& +\sum_{a b r} D_{a}^{r(\lambda)} D_{b}^{r(v)}\left\langle\phi_{a}^{r}|\vec{\nabla}| \phi_{b}^{r}\right\rangle_{\mathbf{r}} \\
& +\sum_{a r s} D_{a}^{r(\lambda)} D_{a}^{s(v)}\left\langle\phi_{a}^{r}|\vec{\nabla}| \phi_{a}^{s}\right\rangle_{\mathbf{r}} \\
& +\sum_{a r} D_{a}^{r(\lambda)} D_{a}^{r(v)}\left\langle\phi_{a}^{r}|\vec{\nabla}| \phi_{a}^{r}\right\rangle_{\mathbf{r}}
\end{aligned}
$$

At the CIS level, the coefficient of the reference configuration $\phi_{0}$ is either one for the ground state or zero otherwise, i.e., $D_{0}^{(\lambda)}=\delta_{0 \lambda}$. Using the Slater-Condon rules to evaluate the integrals, the last term vanishes, and the expression for $\lambda<v$ simplifies to

$$
\begin{aligned}
\int \Phi_{\lambda}^{\mathrm{CIS}} & \left(\mathbf{r}^{N}\right) \vec{\nabla} \Phi_{v}^{\mathrm{CIS}}\left(\mathbf{r}^{N}\right) \mathrm{d} \mathbf{r}^{N-1} \\
= & \sum_{a r} D_{0}^{(\lambda)} D_{a}^{r(v)}\left(\varphi_{a}(\mathbf{r}) \vec{\nabla} \varphi_{r}(\mathbf{r})\right) \\
& +\sum_{a b r} D_{a}^{r(\lambda)} D_{b}^{r(v)}\left(\varphi_{a}(\mathbf{r}) \vec{\nabla} \varphi_{b}(\mathbf{r})\right) \\
& +\sum_{a r s} D_{a}^{r(\lambda)} D_{a}^{s(v)}\left(\varphi_{r}(\mathbf{r}) \vec{\nabla} \varphi_{s}(\mathbf{r})\right) \\
= & \mathbf{j}_{\mathrm{ex}}^{\mathrm{TDCI}}(\mathbf{r}, t)+\mathbf{j}_{\mathrm{h}}^{\mathrm{TDCI}}(\mathbf{r}, t)+\mathbf{j}_{\mathrm{el}}^{\mathrm{TDCI}}(\mathbf{r}, t) .
\end{aligned}
$$

Here, we denote the first term as excitations $\mathbf{j}_{\mathrm{ex}}^{\mathrm{TDCI}}(\mathbf{r}, t)$, the second as hole-hole interactions $\mathbf{j}_{\mathrm{hCI}}^{\mathrm{TDC}}(\mathbf{r}, t)$, and the third as electron-electron interactions $\mathbf{j}_{\mathrm{el}}^{\mathrm{TDCI}}(\mathbf{r}, t)$. This partitioning enables to estimate the contribution of the individual terms during the electron dynamics.

The fundamental relationship between the electron density $\rho(\mathbf{r}, t)$ and flux density $\mathbf{j}(\mathbf{r}, t)$ is the celebrated continuity equation, ${ }^{59}$

$$
\frac{\partial}{\partial t} \rho(\mathbf{r}, t)=-\vec{\nabla} \cdot \mathbf{j}(\mathbf{r}, t) .
$$

From this conservation equation, an associated quantity, the electronic flux, can be derived in terms of the one-electron density. ${ }^{72-74}$ It can be written using the Voronoi scheme introduced above as

$$
F_{V}(t)=\int_{\substack{\text { Voronoi } \\ \text { cell of } V}} \mathrm{~d} \mathbf{r} \frac{\partial \rho(\mathbf{r}, t)}{\partial t} .
$$

This electronic flux gives quantitative information about the net flow of electrons in or out of a selected volume. The time integration of the electronic flux (Eq. (19)) results to the net electronic yield

$$
Y_{V}(t)=\int_{0}^{t} \mathrm{~d} t^{\prime} \int_{\substack{\text { Voronoi } \\ \text { cell of } V}} \mathrm{~d} \mathbf{r} \frac{\partial \rho\left(\mathbf{r}, t^{\prime}\right)}{\partial t^{\prime}}=N_{V}(t)-N_{V}(0),
$$

which specifies the number of electrons that pass through a certain Voronoi volume within a time interval. As a whole, these tools enable a time-resolved analysis of the photoinduced electron migration process, with particular focus on the contribution of different molecular fragments and on the evolution of electrons and holes in the model DSSC.

In this study, all analysis functions, e.g., the electronic yields, and related quantities, e.g., the molecular orbitals, are determined numerically by exploiting the modular structure of our own open-source Python toolbox ORBKIT. ${ }^{58}$ The illustration of grid-based quantities, e.g., the electronic flux density, is performed using the visualization program ZIBAmira. ${ }^{75}$

\section{RESULTS AND DISCUSSION}

\section{A. Characterization of the model system}

In accordance with the description in Section II A, we optimized the geometry of several stoichiometric model clusters with different dissociative binding modes in the gas phase. In the shape of our finite cluster model, the two energetically lowest binding motifs are the 2-M-bidentate bridging and the monodentate attachment of the alizarin to the $\mathrm{TiO}_{2}$ nanocrystallite. All subsequent investigations are carried out for these two model systems. The most stable dye to $\mathrm{TiO}_{2}$ semiconductor binding is the bridgebidentate mode with a total energy difference of $69.0 \mathrm{meV}$ in comparison to the monodentate adsorption. This observation

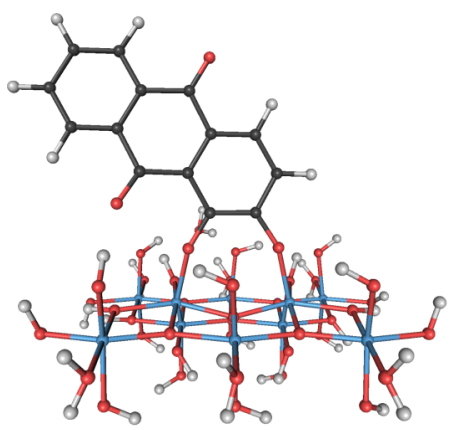

(a)

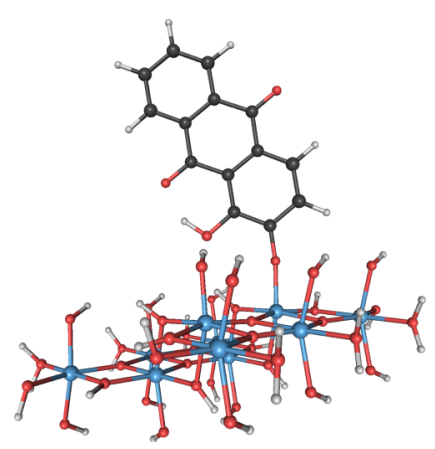

(b)
FIG. 1. Ball-and-stick models of the optimized molecular structures of the alizarin- $\mathrm{TiO}_{2}$ complexes with (a) the bidentate bridging binding motif and with (b) the monodentate binding motif. The dark gray, light gray, red, and cyan beads represent carbon, hydrogen, oxygen, and titanium, respectively. 
can be confirmed by previous studies investigating alizarin$\mathrm{TiO}_{2}$ cluster models. ${ }^{35,76}$ The respective structures for both complexes are illustrated in Figs. 1(a) and 1(b). These figures show that the dye is differently oriented to the nanoparticle in the two systems due to the distinct rigidity of the corresponding binding types. This has an important influence on the electronic coupling and energetic alignment between the dye and the semiconductor. Both characteristics will be seen to affect the time scale and the mechanism of the charge migration process. The influence of dispersion effects is evaluated by performing geometry optimization at the B3LYP-D3 level of theory ${ }^{77}$ to find only minor changes in the molecular structure. This is probably due to the fixation of the $\mathrm{TiO}_{2}$ cluster, which is necessary in order to mimic the structure of a cluster embedded in a thin film. For this constrained structure optimization, the rigidity of dyesubstrate orientation is possibly the origin of the reduced influence of dispersion effects. Dispersion corrections are also found to leave the excitation spectrum unaffected, and they will not be discussed further in the remainder of this paper.

On the basis of the B3LYP reference at the optimized geometry, the optical spectra for both dye-semiconductor model solar cells are computed with linear response TDDFT. The results are depicted in Fig. 2. To verify the adequacy of the computational setting, the main excitation band for the alizarin molecule in its free state is calculated and marked in the spectra (black dashed line) (cf. Fig. 2). As can be observed, the lowest energy band of the free alizarin is centered at $2.84 \mathrm{eV}$, in excellent agreement with the experiment $(2.88 \mathrm{eV}){ }^{36}$ Comparing the two absorption spectra for the monodentate and the bidentate cluster (cf. Fig. 2), two broad bands can be recognized. These exhibit similar energetic positions and oscillator strengths. The lowest energy band is dominated by a single electronic excitation, whereas the second band exhibits numerous excitations. The agreement between the experimental and theoretical spectra is satisfactory, although a moderate blue shift exists $(0.21 \mathrm{eV}$ for the lowest band). As expected for the anchoring of the dye to the $\mathrm{TiO}_{2}$ cluster, the lowest energy band red-shifts by $0.16 \mathrm{eV}$ for both binding types (compared to the experimental shift of $0.41 \mathrm{eV}$ ). Thus, one can conclude that the energy levels between the dye and the semiconductor are properly aligned, and our complexes are suitable models for DSSCs. Interestingly, both model systems have their lowest energy band at $2.68 \mathrm{eV}$ which has also the highest oscillator strength in the spectra. This adsorption band is mainly originated by a HOMO-LUMO transition with a contribution of $92.6 \%$ for the bidentate complex and $97.1 \%$ for the monodentate system. Despite the different orientations of the adsorbate to the $\mathrm{TiO}_{2}$ nanocrystallite, the isosurface plots of these orbitals (cf. Fig. 2) reveal similar nodal structures for both binding motifs. In correspondence with previous theoretical calculations, the HOMO is mostly localized on the carbonyl and hydroxyl groups of the alizarin, whereas the LUMO is evenly distributed on the entire dye..$^{35,38,39}$ Both orbitals also have contributions on the anchoring titanium atoms. Consequently, it can be inferred that the corresponding transitions exhibit a significant intrachromophore chargetransfer character. From a one-electron perspective, the

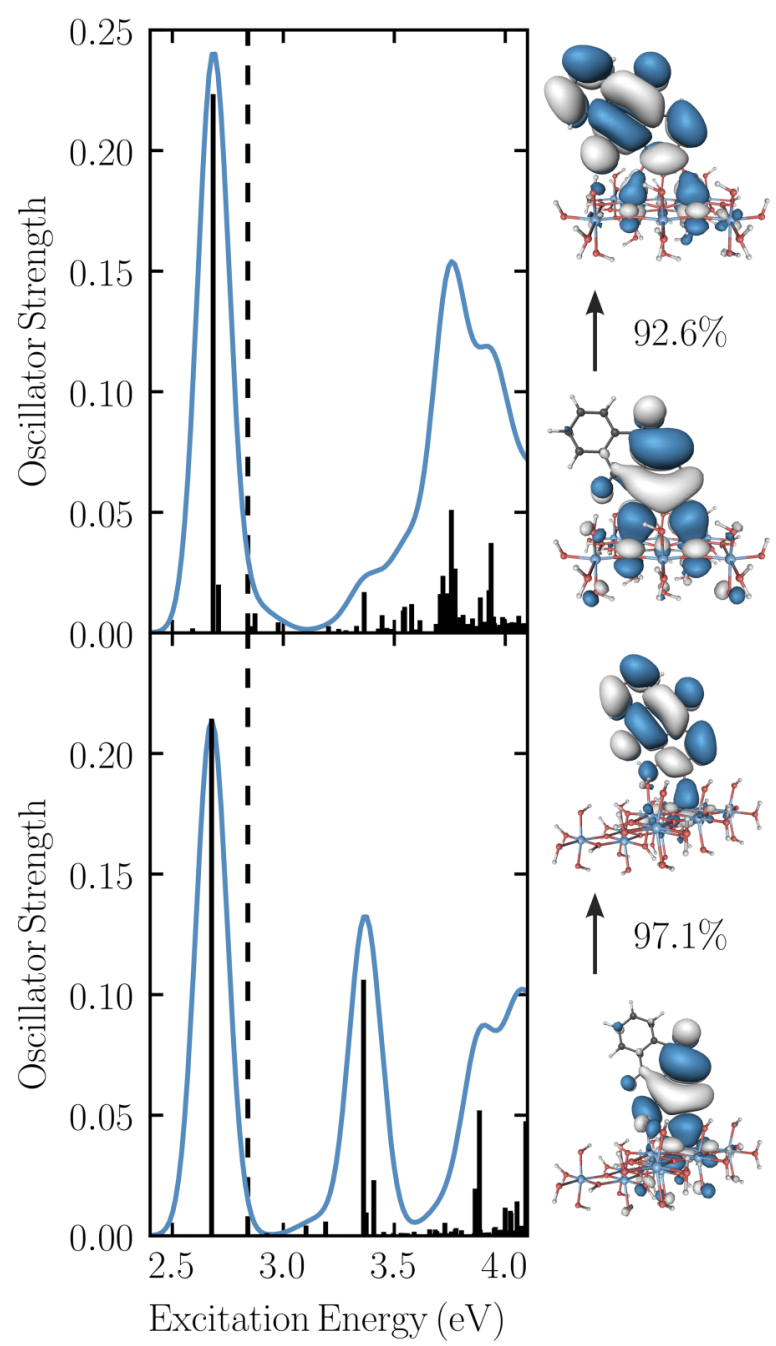

FIG. 2. Simulated optical spectra for alizarin- $\mathrm{TiO}_{2}$ system with bidentate binding type (upper panel) and with monodentate anchoring mode (lower panel) obtained from linear response TDDFT calculations with B3LYP functional. Vertical black lines signify the oscillator strengths of the specific transitions. In the experimental absorption spectra of alizarin- $\mathrm{Ti}_{2} \mathrm{O}$ complexes, there are two main bands centered at $2.47 \mathrm{eV}$ and $3.55 \mathrm{eV}$. The position of the theoretical band of the free alizarin is marked as a dashed black line at $2.84 \mathrm{eV}$. The associated experimental absorption band is centered at $2.88 \mathrm{eV}$. The broadened spectra (solid blue lines) are constructed using Gaussian functions with a width of $\sigma=0.1 \mathrm{eV}$. For the main absorption band at $2.68 \mathrm{eV}$ appearing in both systems, isosurface plots of the dominant frontier orbitals (HOMO, LUMO) are depicted with the associated transition contribution. The isosurface value is set to $\pm 10^{-2} \mathrm{a}_{0}^{-3 / 2}$.

electron transfer process is initiated from the HOMO-LUMO photoexcitation. This creates an electron-hole pair followed by the migration of the excited electron from the LUMO to the $\mathrm{TiO}_{2}$ cluster. From this perspective, it appears that a single active electron approach (TDKS) (cf. Sec. II), including only the HOMO for the initial state and a set of virtual orbitals in the wave function expansion for the active electron, will provide a good simplification of the many-electron TDCI approach.

For the photoexcitation, a comparative analysis of the static electronic structure, described for both theoretical approaches and for both model systems, is performed using a set of density-based diagnostic tools developed by Ciofini and co-workers ${ }^{78,79}$ to study charge-transfer excitations in 
(a)

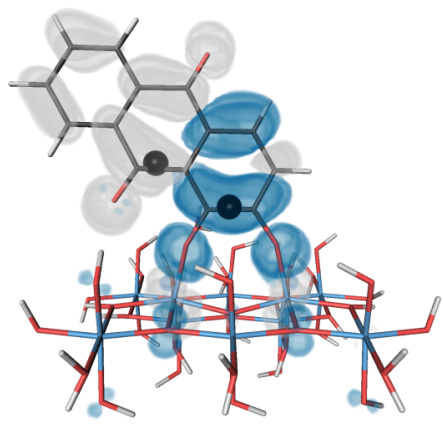

(b)

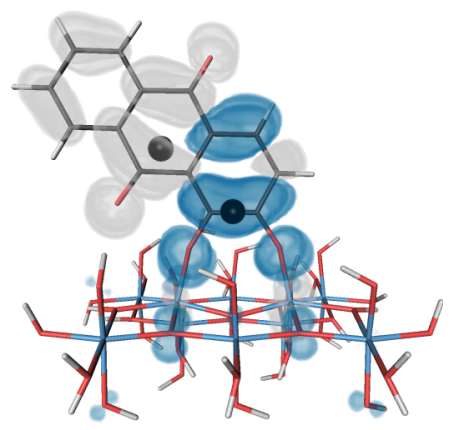

(c)

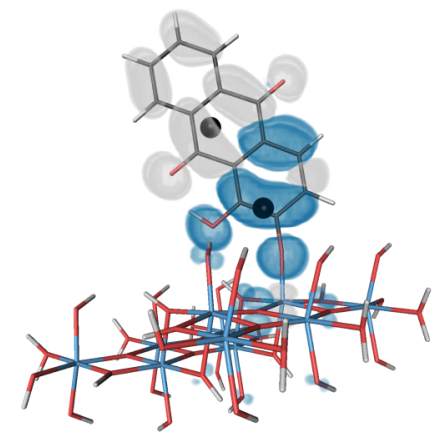

FIG. 3. Isosurface plots of the difference density $\left(\Delta \rho(\mathbf{r})=\rho_{\mathrm{t}}(\mathbf{r})-\rho_{\mathrm{g}}(\mathbf{r})\right)$ for the alizarin- $\mathrm{TiO}_{2}$ complex with 1,2-bidentate binding on the basis of (a) the many-body wave function $(\mathrm{CI})$ and (b) the single-particle wave function (KS), and for (c) the alizarin- $\mathrm{TiO}_{2}$ system with monodentate attachment within the single active electron approach. The molecular structure is represented as a stick model. The atomic species $\mathrm{C}, \mathrm{H}, \mathrm{O}$, and Ti are colored dark gray, light gray, red, and cyan, respectively. The negative isosurface (blue) corresponds to $\rho_{-}$with a threshold of $-10^{-3} \mathrm{a}_{0}^{-3}$ and the positive isosurface (gray) indicates $\rho_{+}$with a threshold of $10^{-3} \mathrm{a}_{0}^{-3}$. The respective barycenters are marked with black balls.
DSSCs. First, the difference density between the B3LYP ground state and the dominant excited state wave function contribution to the main absorption band (target state), $\Delta \rho(\mathbf{r})=\rho_{\mathrm{t}}(\mathbf{r})-\rho_{\mathrm{g}}(\mathbf{r})$, is computed. Note that this simplifies to the HOMO-LUMO transition in the single active electron ansatz. The position of the barycenters for the density depletion $(\Delta \rho(\mathbf{r})<0)$ and density concentration regions $(\Delta \rho(\mathbf{r})>0)$ is determined by integrating the difference density over negative or positive values, respectively. These centers of charge simplify determination of the donor and acceptor groups in charge-transfer complexes.

The difference densities and barycenters are shown in Fig. 3. Panels (a) and (b) provide a comparison between the many-body (CI) and one-particle (KS) approaches for the bidentate system, while panels (b) and (c) shed light on the differences between the two anchoring types within the single active electron ansatz. In general, the difference densities and the position of the barycenters appear qualitatively similar for both systems and both using both approaches. Consequently, it can be deduced that the electron densities are hardly affected by the many-body character of the wave function. The character of the difference density in the many-body approach (CI) chiefly arises from the high contribution of the HOMOLUMO transition. From Fig. 3, both barycenters are seen to be located on the dye, thereby confirming the intrachromophore character for the excitation from the ground state to the target state. The barycenter of the density enhancement (gray contour) is positioned in the central ring of alizarin, while the barycenter of the density depletion (blue contour) is positioned near the $\mathrm{TiO}_{2}$ substrate. Consequently, the lone pairs of the carbonyl groups can be indicated as electron donor and the $\mathrm{TiO}_{2}$ cluster as electron acceptor after the excitation.

To supplement this visual analysis, Table I reports the distance between the barycenters $D_{\mathrm{CT}}$, which is referred to as charge transfer length, the transferred charge $\left(q_{\mathrm{CT}}\right.$ $\left.=\int \mathrm{d} \mathbf{r} \rho_{+}(\mathbf{r})\right)$, and the transition dipole moment between the ground and target state $\left(\mu_{\mathrm{CT}}=D_{\mathrm{CT}} \int \mathrm{d} \mathbf{r} \rho_{+}(\mathbf{r})\right)$. It is seen that both methods are in quantitative agreement for the charge transfer, while the charge transfer length is slightly overestimated in both complexes at the one-electron level of theory. Consequently, the transition dipole moment is also found to be marginally larger in the one-electron ansatz, which will mostly influence the excitation efficiency. As a result, we can conclude that the one-electron approach is sufficient to properly characterize the static properties of the model systems. Interestingly, the fraction of transferred charge within the chromophore, $q_{\mathrm{CT}}$, is seen to be insensitive to the binding type, but the charge transfer length is distinctly heightened for the monodentate mode. Here, the different orientation of the chromophore to the $\mathrm{TiO}_{2}$ cluster impacts the hybridization of the dye and substrate orbitals, which leads to a different distribution of the electron density at the interface, see Figs. 3(b) and 3(c). It is seen that the bidentate bonding mode provides a better overlap of the orbital with the substrate than the monodentate species. As a consequence, a

TABLE I. Comparison of the distance between the barycenters $D_{\mathrm{CT}}$, the transferred charge $q_{\mathrm{CT}}$, and the transition dipole moment between the ground and the target state $\mu_{\mathrm{CT}}$ for the two bonding modes at different levels of theory, many-body approach (CI) and single active electron ansatz (KS).

\begin{tabular}{|c|c|c|c|c|}
\hline \multirow{2}{*}{$\frac{\text { Binding type }}{\text { Approach }}$} & \multicolumn{2}{|c|}{ Bidentate } & \multicolumn{2}{|c|}{ Monodentate } \\
\hline & $\mathrm{CI}$ & $\mathrm{KS}$ & CI & KS \\
\hline$D_{\mathrm{CT}}(\AA)$ & 2.67 & 2.97 & 3.51 & 3.62 \\
\hline$q_{\mathrm{CT}}\left(\mathrm{e}^{-}\right)$ & 0.68 & 0.70 & 0.71 & 0.70 \\
\hline$\mu_{\mathrm{CT}}(\mathrm{D})$ & 8.75 & 10.06 & 11.99 & 12.17 \\
\hline
\end{tabular}


much slower injection dynamics can be expected in the latter case, as will be confirmed in Sec. III B.

\section{B. Electron-transfer dynamics}

In the following, the photoinduced electron transfer dynamics from the alizarin to the $\mathrm{TiO}_{2}$ cluster is studied in real time for the bidentate and monodentate alizarin- $\mathrm{TiO}_{2}$ cluster (cf. Figs. 1(a) and 1(b), respectively). One focus of the dynamical study is set on the comparison between the timedependent single active electron ansatz, TDKS, and the timedependent many-electron configuration interaction approach, TDCI. All simulations are performed using an in-house implementation of the $\rho$-TDCI method, ${ }^{28,61}$ incorporating the ground state and the first ten excited states in the dynamical basis at the respective level of theory.

In general, the overall dynamical process starts with the photoexcitation of the main absorption band in the TDDFT spectra (cf. Fig. 2) and proceeds subsequently with the charge migration from the dye to the semiconductor. For the laserinduced excitation, we tailor the parameters of a $\sin ^{2}$-shaped $\pi$-pulse (cf. Eq. (3)) to the experimentally applied laser pulse in transient absorption measurements in similar systems. ${ }^{11,42}$ Here, a pulse of 19 fs duration polarized normal to the $\mathrm{TiO}_{2}$ cluster is used, followed by $20 \mathrm{fs}$ of free decay. In the subsequent laser-driven dynamics, all pulse frequencies are tuned at the respective transition energies. With respect to the TDDFT results (cf. Fig. 2), the TDCI simulation is initiated by excitation from the ground state $\Phi_{\mathrm{g}}$ to the second excited state for the bidentate complex and to the first excited state for the monodentate system. These charge transfer states are referred to as the target state $\Phi_{\mathrm{t}}$. In contrast, the HOMOLUMO transition is promoted in the TDKS propagations. As described in Section II, the initial wave function in the TDCI method is formed by many-body configuration interaction functions (cf. Eq. (6)) based on a TDDFT calculation. To ensure that the time scales are comparable using the TDCI and TDKS approaches, the band gap at the KS level of theory is simply corrected by shifting the energy levels of the virtual orbitals to the main absorption band of the corresponding TDDFT spectrum.

A crucial component of the dynamical simulation is the absorbing potential (cf. Eq. (9) for TDKS and Eq. (10) for TDCI), which prevents artificial reflection of the electronic wave packet at the edges of the $\mathrm{TiO}_{2}$ cluster. This should be understood as an intrinsic property of the cluster embedded in the thin film, and it should be independent of the dye itself. Consequently, the injection rate is adjusted to reproduce the experimental rate for alizarin adsorbed on $\mathrm{TiO}_{2}$ thin films $(1 /(60 \mathrm{fs})) .{ }^{42}$ The reference is chosen as the energetically most stable bonding motif, the bidentate cluster, as it is presumably more abundant in the experiment and would thus dominate the injection dynamics. The CI reference state $\Phi_{t}$ is identified by inspection of the dominant configuration state function to match the dominant orbital contribution of the KS ansatz. For the reference state $\Phi_{\mathrm{t}}$, the injection rate is thus the same at both levels of theory. This corresponds to a scaling factor of $\gamma_{\mathrm{CAP}}=6.6 \mathrm{fs}^{-1}$ for the TDCI ansatz and of $\gamma_{\mathrm{CAP}}=10.6 \mathrm{fs}^{-1}$ for the TDKS approach. The latter is also used for the less stable monodentate system. As a result, the relaxation rate for the bidentate complex $(1 /(60 \mathrm{fs})$ for TDKS) is one order of magnitude faster than the one for the monodentate cluster $(1 /(474 \mathrm{fs})$ for TDKS). In general, scaling of the relaxation rates of the absorbing potential is essential to suppress spurious, competing processes that are slower than electron injection.

The time-evolution of the state populations is depicted in Fig. 4(a), while the laser-field and the expectation values of the dipole moment are depicted in Fig. 4(b). To allow comparison between the distinct theoretical approaches, the population
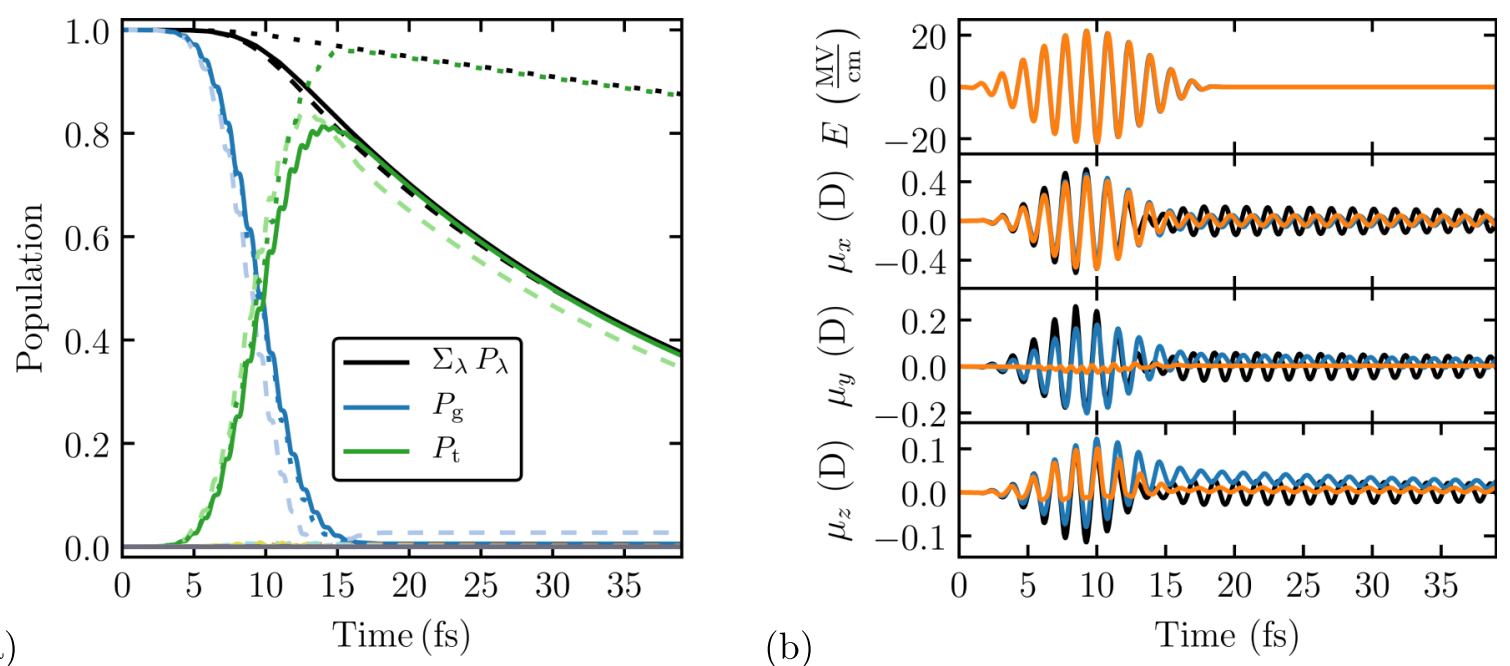

FIG. 4. Laser-driven electron transfer dynamics for the differently attached alizarin/ $\mathrm{TiO}_{2}$ model clusters at the TDCI and TDKS levels of theory. The systems are excited using a $\sin ^{2} \pi$-laser pulse with a pulse duration of $19 \mathrm{fs}$, followed by $20 \mathrm{fs}$ of free decay. (a) Comparison of the population evolution for the bidentate binding mode within the TDCI approach (dashed lines) and the TDKS ansatz (solid lines), and for the monodentate attachment motif within the TDKS approach (dotted lines). The populations of the ground state $P_{\mathrm{g}}$ and the target state $\boldsymbol{P}_{\mathrm{t}}$ of the laser excitation are depicted as blue and green lines, respectively. (b) Electric field (upper panel) and dipole moment expectation values (lower three panels) for the different binding mode and approach combinations. These are colored black for bidentate/TDCI, blue for bidentate/TDKS, and orange for monodentate/TDKS. The ground state and ten excited states are incorporated in the simulations. 
evolution for the interface with the bidentate binding type is depicted within the TDCI ansatz (cf. Fig. 4(a) (dashed lines)) and the TDKS approach (cf. Fig. 4(a) (solid lines)). As can be noticed, both theoretical approaches yield quantitatively similar results, with an initial localized dye-dye excitation during the laser pulse, followed by an apparently monoexponential decay of the target state. The latter time scale appears not to be affected by the correlated TDCI wave packet. Since neither the excitation nor the population decay is significantly affected by the level of theoretical description, we can deduce that the many-body character of the TDCI wave function plays a subordinate role to the population dynamics in our model system. This is conceivably due to the fact that the dynamical processes associated with manybody character are on significantly longer time-scales than the electron migration. As a consequence of the applied absorbing boundary conditions, these processes are suppressed in our electron dynamics.

To detect the differences between the binding types, the bidentate system is compared with the monodentate complex (cf. Fig. 4(a) (dotted line)) at the TDKS level. A slower relaxation rate $(1 /(474 \mathrm{fs}))$ of the target state population $P_{\mathrm{t}}$ for the monodentate system can be observed, which is caused by the smaller overlap of the target excited state with the absorbing potential at the edges of the cluster. As discussed in Sec. III A, the orbitals of the monodentate species are only weakly hybridized with those of the semiconductor, and their penetration in the substrate is reduced. In general, all three simulations exhibit a similar course of the population evolution, in which solely the ground state and the target excited state are significantly populated. This suggests that the photoexcited electron is instantaneously absorbed at the boundary of the $\mathrm{TiO}_{2}$ nanoparticle. The excited states which are delocalized onto the semiconductor, only serve as mediator for the charge migration.

The evolution of the dipole moments underlines the similarities of both methods. In all three simulations, all components first strongly react to the laser-pulse, while rapid oscillations are observed after the field is switched off. In all cases, the creation of an electronic wave packet as a superposition of the ground state and the photoexcited state is responsible for the observed Rabi oscillation in the dipole moment. The period of these oscillations corresponds perfectly to the transition energy, $\tau=h / \Delta E=h / 2.68 \mathrm{eV}=1.54 \mathrm{fs}$. For the bidentate system, the disparities between the TDCI approach (cf. Fig. 4(b) (black line)) and the TDKS ansatz (cf. Fig. 4(b) (blue line)) are marginal during the pulse. Some minor discrepancies (e.g., components out of phase or with different amplitudes) are observed after the pulse, in particular for the components perpendicular to the laser polarization ( $y$ and $z$ ), but the qualitative behavior remains well reproduced at the TDKS level. This hints that the many-electron character plays only a minor role in the injection process of alizarin on titania.

More surprisingly, the oscillation periods observed from the TDKS simulation for the bidentate and monodentate species along the $x$ axis coincide perfectly. This is in part due to the identical excitation energies $(2.68 \mathrm{eV})$, and in part to the similar nodal structure of the HOMO and LUMO (cf. Fig. 2). This gives rise to a dipole normal to the surface (i.e., along the $x$ axis) of about the same magnitude in both cases. The $z$-component of the dipole moment (lowest panel) has a qualitatively similar behavior for both bonding motifs, while the $y$-dipole remains unaffected for the monodentate species. This difference can be attributed to the orientation of the alizarin on the model clusters, as depicted in Fig. 1.

\section{Electron flow and electron/hole correlation}

Understanding the flow of electrons can provide important mechanistic insight into the charge migration process. For this purpose, we make use of the time-dependent electronic yields $Y_{V}(t)$ (cf. Eq. (20)), the electronic fluxes $F_{V}(t)$ (cf. Eq. (19)), and the electronic flux densities $\mathbf{j}(\mathbf{r}, t)$ (cf. Eq. (13)). For the space-resolved analysis of the dynamical simulations, a Voronoi partitioning scheme of the space belonging to the alizarin donor and the semiconductor is advocated (cf. Sec. II). To start with, the electronic yields and fluxes are compared between the TDKS approach and the TDCI method for the alizarin- $\mathrm{TiO}_{2}$ cluster with the bidentate bonding motif. Fig. 5 shows the evolution of the electronic yields $Y_{V}(t)$ (lower panel) and fluxes (two upper panels) after the excitation with the laser-pulse for four different Voronoi cells: the complete alizarin molecule, the semiconductor substrate, and the two carbonyl groups of the dye. The latter were shown to play an important role in the injection process. ${ }^{25}$ Here, we label the carbonyl group that faces away from the $\mathrm{TiO}_{2}$ cluster as "top CO" group, and the other one as "bottom CO" group. The most obvious difference in Fig. 5 is the oscillatory pattern of the electronic yields within the TDKS approach, whereas the results for the TDCI ansatz exhibit almost smooth

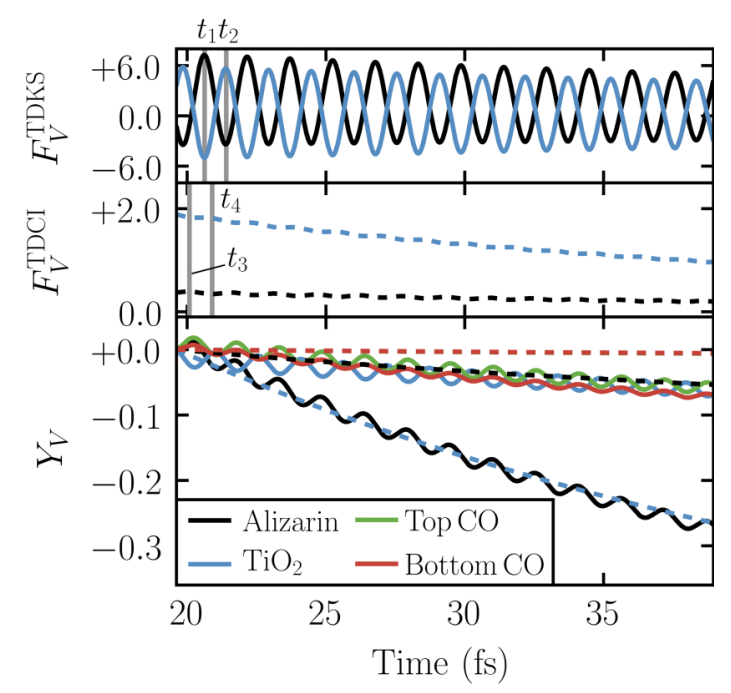

FIG. 5. Time evolution of the electronic yields $Y_{V}(t)$ and electronic fluxes $F_{V}(t)$ during the charge migration dynamics from the alizarin towards the $\mathrm{TiO}_{2}$ nanocrystallite after photoexcitation. Upper panel: electronic flux for TDKS (solid lines) (in units of $10^{-2} \mathrm{fs}^{-1}$ ). Central panel: electronic flux for TDCI (dashed lines) (in units of $10^{-2} \mathrm{fs}^{-1}$ ). Lower panel: electronic yields. The fluxes and yields are determined for different Voronoi polyhedrons, i.e., the alizarin dye (black), the $\mathrm{TiO}_{2}$ cluster (blue), the top carbonyl group (green), and the bottom carbonyl group (red). 
decaying curves. This behavior can be explained by the different constructions of the initial wave functions for both approaches (cf. Eqs. (6) and (7)). On the one hand, the initial one-electron density is constructed from an $\mathrm{N}$-electron wave function in the TDCI method, and is thus equally distributed over the whole dye-semiconductor cluster. On the other hand, a one-electron wave function corresponding to the HOMO is used as an initial state in the TDKS propagation. In this case, the initial one-electron density is only localized on the alizarin and on the anchoring titanium atoms (cf. Fig. 2). Since the one-electron densities in both methods are normalized, the fluctuations of the time-dependent densities are small in the TDCI ansatz in comparison to the TDKS approach.

Turning our attention to the contributions of the different Voronoi cells of the electronic yields (cf. lower panel of Fig. 5), the initial density distribution for the correlated ansatz appears to be more delocalized on the titanium cluster. This is evidenced by the larger relative yield of the latter (dashed blue line), which is on the same order as that of the alizarin donor in the TDKS approach. Further, the one-electron ansatz

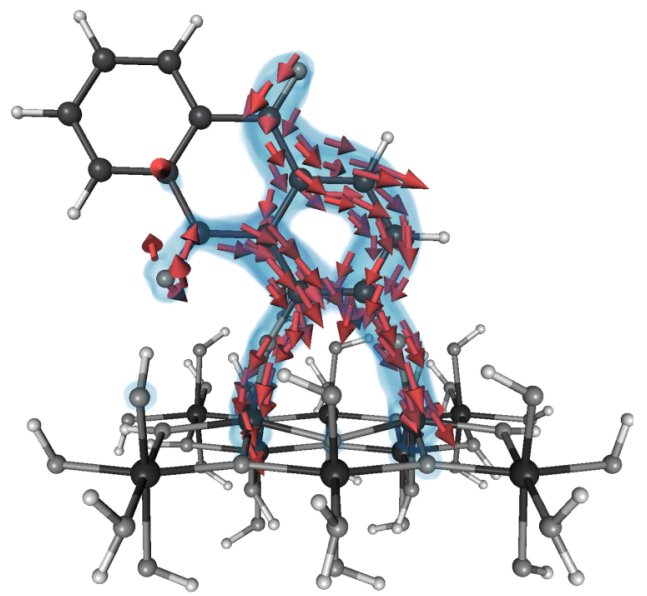

$$
t_{1}=20.63 \mathrm{fS}
$$

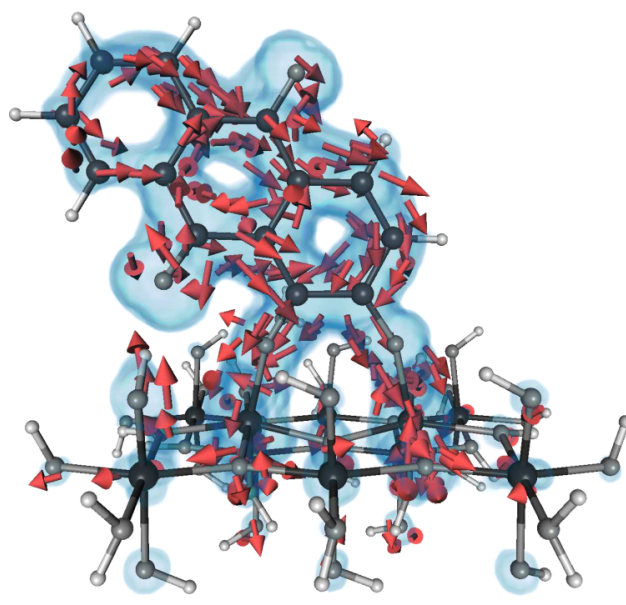

$t_{3}=20.08 \mathrm{fs}$ shows larger oscillations in the yields, with the dye and the semiconductor contributions of opposite phase, as is the case for the associated fluxes (cf. upper panel of Fig. 5). Nonetheless, an alternating charge migration process between the dye and the semiconductor emerges for TDCI, albeit of smaller amplitude. Interestingly, the contributions of the top and bottom $\mathrm{CO}$ groups (red and green lines) are in phase, but they are larger for the one-electron than for the correlated ansatz. The former also appear to be asymmetric with respect to the injection yield. These marginal differences hint at a small influence of the many-electron nature of the wave packet, an effect which will be investigated in the following.

To provide a more detailed picture of the instantaneous flow of the electron density, the electronic flux densities $\mathbf{j}(\mathbf{r}, t)$ and their magnitudes are illustrated for both methodologies in Fig. 6 at selected times. The snapshots are selected at the turning points of the dynamical simulations, where the charge migrates from the alizarin to the substrate and vice versa (vertical gray lines in the upper and central panels of Fig. 5). The flux density is a vector field that specifies the
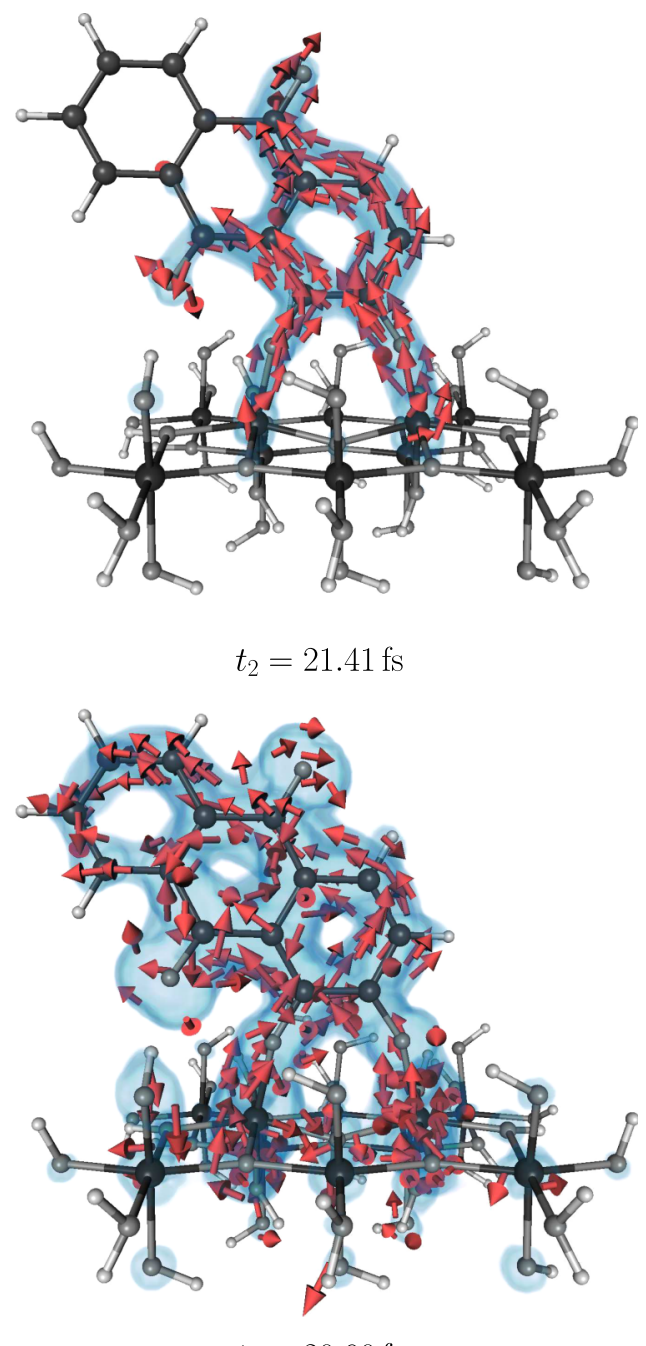

$t_{4}=20.90 \mathrm{fs}$

FIG. 6. Vector plots of the electronic flux density $\mathbf{j}(\mathbf{r}, t)$ and its magnitude as a isosurface plot at selected times. The points in time are marked as gray lines in Fig. 5. These are chosen as extrema of the electronic flux, which coincide with turning points in the dynamics. The upper (lower) panels show the results at the TDKS (TDCI) level of theory. The contour value for the magnitudes is defined as $10^{-4} E_{\mathrm{h}} / \hbar a_{0}^{2}$. 
flow of electrons at every point in space and is consequently independent of a partitioning scheme. The magnitudes of the electronic flux density, $|\mathbf{j}(\mathbf{r}, t)|$, are also depicted to reveal its spatial distribution. For both approaches, the flux densities show some common characteristics: (1) the electrons flow along the bonds on the aromatic dye and at the Ti-O anchoring points; (2) synchronous fluxes towards the CO groups and the other way around at different time steps; (3) the close-lying hydroxyl groups at the surface can serve as bridges between the dye and the substrate; and (4) the main spatial distribution of flux density is located on the dye and anchoring titanium atoms. These findings are in line with previous work. ${ }^{25}$ The second point can be likewise confirmed from the electronic yields in Fig. 5 (upper panel), showing simultaneous oscillations of both $\mathrm{CO}$ groups. Apart from these similarities, the electronic flux density within the TDCI ansatz appears slightly more complex. Whereas the one-electron approach predicts that only the bottom part of the dye participates in the injection process, the mechanism at the many-electron level of theory involves the whole molecule. The TDKS flux density shows that electrons follow two channels: the top and the bottom CO contributions barely mix, and the associated electrons migrate simultaneously to the nearest $\mathrm{Ti}-\mathrm{O}$ anchoring bond. The bottom part of the TDCI flux density behaves somewhat similarly, but the topmost ring exhibits a directional ring current that correlates with the phase of the migration process. During the injection phase (bottom right panel, $t_{3}$ ), the hydroxy group at the surface donates electrons back to the bottom carbonyl via the hydrogen bond. Consequently, this electron rich carbonyl transfers electron to the top $\mathrm{CO}$ via the topmost ring. During the backward reaction (bottom left panel, $t_{4}$ ), the hydrogen bond is only weakly involved, and the hydroxy group donates its electrons preferentially to the surface, towards neighboring Ti-O anchoring group. The bottom $\mathrm{CO}$ becomes electron poor, and the ring current has an opposite sign. Since both the nature of the injection state in the TDKS and $\mathrm{N}$-electron approaches are similar (92.6\% HOMO-LUMO character for TDCI), this distinctive behavior stems from the small contributions of the singly excited configuration state function with excitations from core MOs.

In order to acquire a better understanding of the origin of the differences between both methods, the electron yield and the associated flux density at the TDCI level are computed using one-electron wave functions. To this end, each state obtained from linear response TDDFT forming the basis for the TDCI calculation is associated to a single MO, with the ground state defined as the HOMO. Note that the timedependence of the expansion coefficients of the wave function is taken from the fully correlated propagation, and is therefore correlated. Only the flux density analysis is modified, and the one-electron wave packet (cf. Eq. (7)) in this so-called correlated TDKS takes the form

$$
\Phi_{n}^{\mathrm{cTDKS}}(\mathbf{r})=\sum_{\lambda} D_{\mathrm{HOMO}}^{n(\lambda)} \varphi_{n}(\mathbf{r}) .
$$

The time evolution of electronic yields and a snapshot of the electronic flux density for this correlated TDKS ansatz can
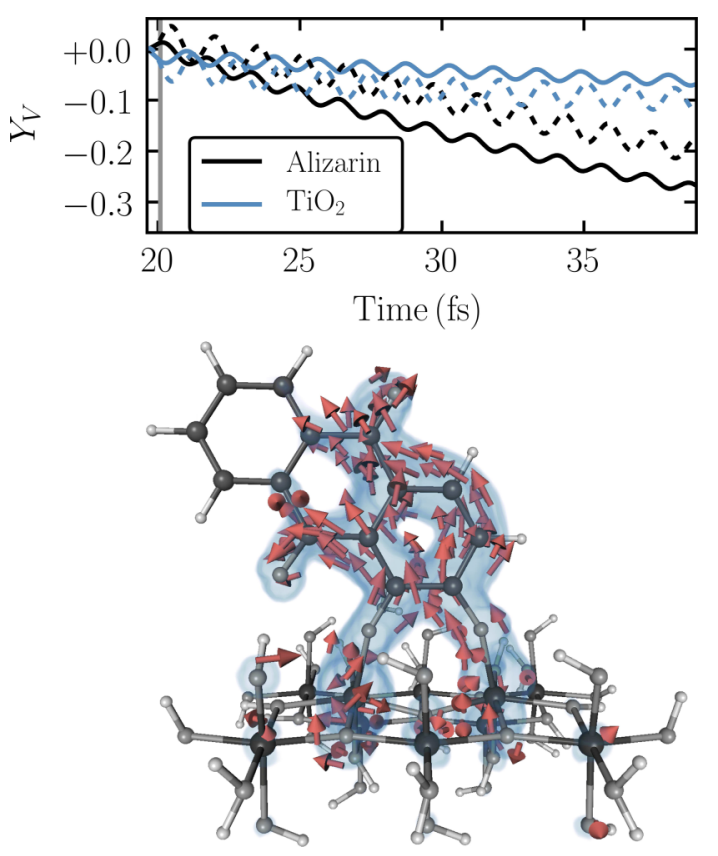

FIG. 7. Evolution of the electronic yields and snapshot of the electronic flux density. The yields are spatially partitioned with the Voronoi scheme into the alizarin dye and the $\mathrm{TiO}_{2}$ cluster. The alizarin bidentate attaching mode to the $\mathrm{TiO}_{2}$ substrate is depicted within the correlated TDKS method (Eq. (21), dashed lines) and at the TDKS level (solid lines). The contour value for the flux density magnitudes is defined as $10^{-4} E_{\mathrm{h}} / \hbar a_{0}^{2}$. A gray line marks the point in time for the flux density snapshot.

be seen in Fig. 7 (dashed lines). It can be seen that both the electronic yields and the electronic flux density show a qualitatively good agreement with the results of the pure

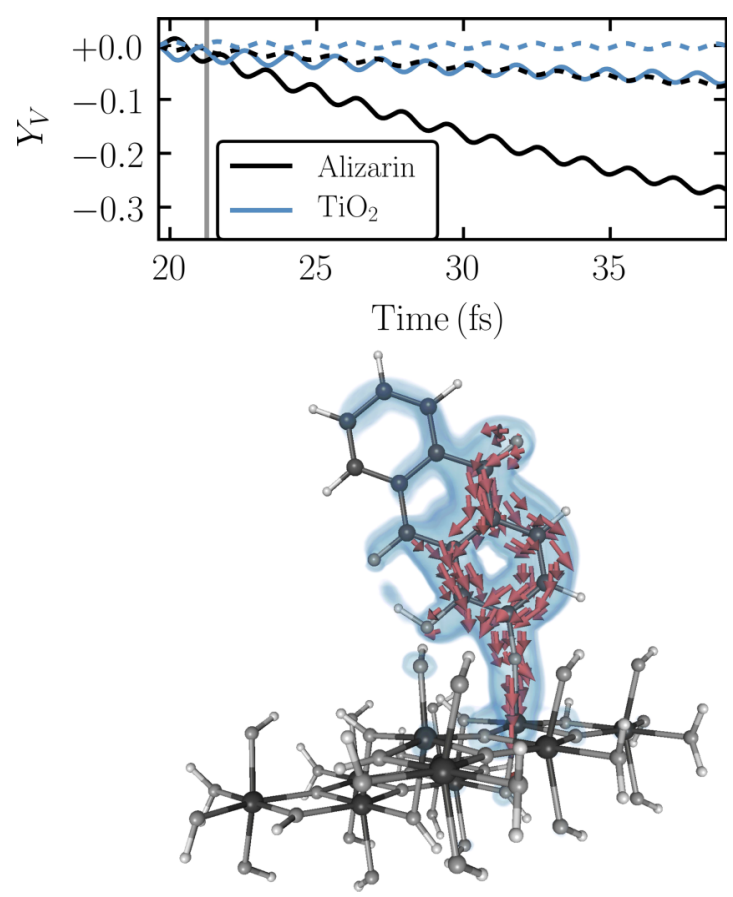

FIG. 8. Evolution of the electronic yields for bidentate (solid lines) and monodentate (dashed lines) anchoring motifs. The snapshot of the electronic flux density corresponds to the alizarin- $\mathrm{TiO}_{2}$ cluster with monodentate binding type at the TDKS level. The contour value for the flux density magnitudes is defined as $2.5 \times 10^{-5} E_{\mathrm{h}} / \hbar a_{0}^{2}$. A gray line marks the point in time for the flux density snapshot. 
TDKS method (solid lines). The discrepancies between the time scales and the yields stem from the different initial conditions after the pulse, which is more efficient at the TDKS level of theory. The correlation of the expansion during the dynamics appears otherwise not to affect the migration mechanism. The qualitative differences in Fig. 6 are thus solely due to the correlated nature of the basis used to represent the $N$-electron wave packet.

For comparison purposes, Fig. 8 shows the time evolution of electronic yields for the bidentate (solid) and monodentate (dashed) anchoring types, at the TDKS level of theory. The coherent behavior is largely similar in both cases due to simplicity of the excitation mechanism - the created wave packet is mostly composed of only two components at the same transition energy. A similar oscillating behavior of the electronic yields is observed, i.e., opposite phase of the alizarin to the $\mathrm{TiO}_{2}$ cluster. It comes as no surprise that the decay of the yields is weaker for the monodentate species due to the significantly lower hybridization and reduced penetration of the dye orbitals with the semiconductor. The mechanism reveals many interesting novel features. First, as observed for the bidentate case (cf. Fig. 6), the top CO is strongly involved in the injection process. On the other hand, electrons originating from the top carbonyl flow concertedly on the bottom ring. The rightmost electrons are then injected in the substrate via the $\mathrm{Ti}-\mathrm{O}$ bond, while electrons along the left path migrate to the $\mathrm{OH}$ group not bound to the surface. This electron rich hydroxyl group is found to build a weak hydrogen bridge with the bottom carbonyl group rather than with the surface, as it is the case for bidentate species. Note that these mechanistic features do not improve the injection efficiency, which remains one order of magnitude slower and, as such, is only a minor channel in the DSSCs of this type.

As a last aspect in our analysis, the electronic flux density in the TDCI approach is decomposed to evaluate the contribution of electron-electron and hole-hole interactions in the DSSCs during the dynamics. To this end, the corresponding flux densities $\mathbf{j}_{\mathrm{el}}^{\mathrm{TDCI}}(\mathbf{r}, t)$ and $\mathbf{j}_{\mathrm{h}}^{\mathrm{TDCI}}(\mathbf{r}, t)$ are computed using the definitions in Eq. (17). In Figs. 9(a) and 9(b), illuminated streamline ${ }^{80}$ plots of the flux densities are shown together with contour plots of the respective magnitudes during a free decay of $50 \mathrm{fs}$. From the spatial distribution of the flux density components and their magnitudes, it can be deduced (a)

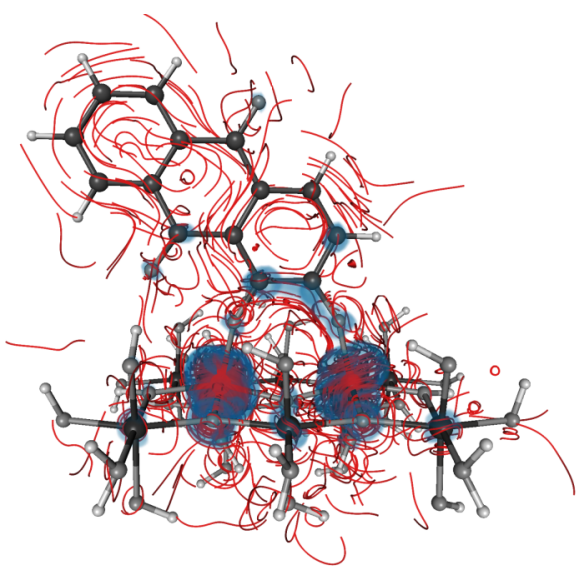

(b)

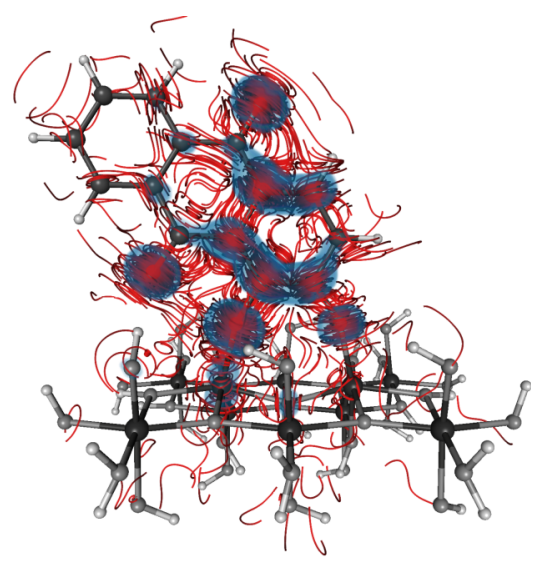

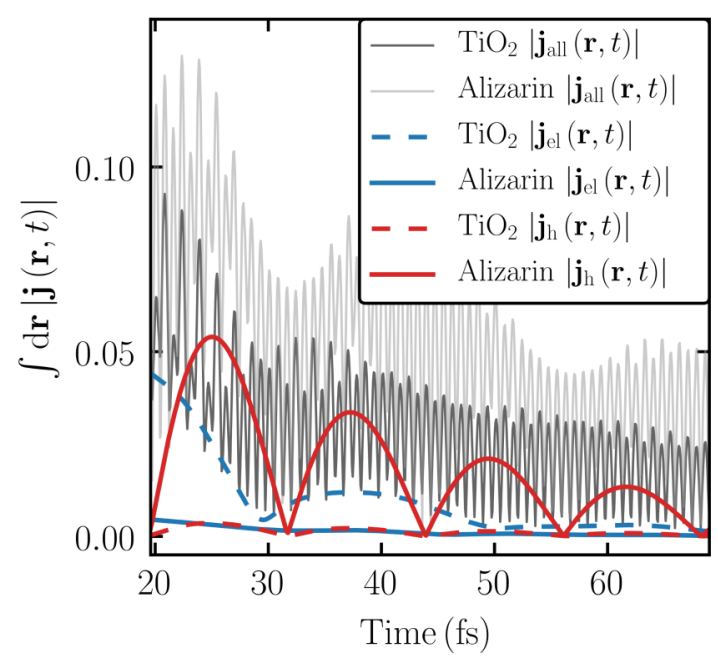

FIG. 9. Electronic flux density $\mathbf{j}(\mathbf{r}, t)$ for the solar cell with bidentate binding mode decomposed for (a) electron-electron and (b) hole-hole interactions within the TDCI approach. Illuminated streamline plots of $\mathbf{j}_{\mathrm{el}}^{\mathrm{TDCI}}(\mathbf{r}, t)$ and $\mathbf{j}_{\mathrm{h}}^{\mathrm{TDCI}}(\mathbf{r}, t)$ and isosurface plots of their magnitudes illustrate the spatial distribution. The contour value is set to $7.5 \times 10^{-5} E_{\mathrm{h}} / \hbar a_{0}^{2}$. (c) Time-evolution of the magnitudes of the electronic flux density $\int \mathrm{d} \mathbf{r}|\mathbf{j}(\mathbf{r}, t)|$ integrated over the different Voronoi cells (in units of $E_{\mathrm{h}} a_{0} / \hbar$ ), alizarin molecule and semiconductor substrate. This quantity is calculated for total electronic flux density (gray lines), as well as for its electron-electron (blue lines) and hole-hole contributions (red lines). 
that the electron-electron component $\mathbf{j}_{\mathrm{el}}^{\mathrm{TDCI}}(\mathbf{r}, t)$ is dominantly localized around the anchoring titanium atoms, whereas holehole correlation $\mathbf{j}_{\mathrm{h}}^{\mathrm{TDCI}}(\mathbf{r}, t)$ of the flux density resides at the dye. This novel dynamical quantity complements the static analysis (cf. Sec. II A): whereas the hole-hole correlation is located at the barycenter, the electron-electron correlation is centered at the anchoring $\mathrm{Ti}$ atoms, far from the corresponding barycenter. Interestingly, while both hole-hole and electronelectron correlations are more localized on the atoms than on the bonds because they bear most of the electron density, these far-reaching correlations spread over the whole dye.

Using Voronoi partitioning, the magnitudes of the vector fields are spatially decomposed for alizarin and the $\mathrm{TiO}_{2}$ domain. The results of this partitioning for the total electronic flux density $\mathbf{j}^{\mathrm{TDCI}}(\mathbf{r}, t)$ (gray lines), the electronelectron correlation $\mathbf{j}_{\mathrm{el}}^{\mathrm{TDCI}}(\mathbf{r}, t)$ (blue lines), and hole-hole component $\mathbf{j}_{\mathrm{h}}^{\mathrm{TDCI}}(\mathbf{r}, t)$ (red lines) are reported in Fig. 9(c). Besides confirmation of the spatial distribution of the single contributions, this analysis allows identifying different time scales for the fluctuations of the various flux densities. In particular, it is found that $\mathbf{j}_{\mathrm{el}}^{\mathrm{TDCI}}(\mathbf{r}, t)$ (blue lines) and $\mathbf{j}_{\mathrm{h}}^{\mathrm{TDCI}}(\mathbf{r}, t)$ (red lines) oscillate much more slowly than the total flux density $\mathbf{j}^{\mathrm{TDCI}}(\mathbf{r}, t)$ (gray lines). From the definition of $\mathbf{j}_{\mathrm{el}}^{\mathrm{TDCI}}(\mathbf{r}, t)$ and $\mathbf{j}_{\mathrm{h}}^{\mathrm{TDCI}}(\mathbf{r}, t)$ in Eq. (17), it is evident that both quantities exclusively arise from excited states. Due to their dense energetic distribution $\left(\Delta E_{1,11}=0.74 \mathrm{eV}\right)$, we see these slow oscillations of the magnitudes in Fig. 9. Accordingly, the total electronic flux density appears to be dominated by excitations from the ground state $\left(\Delta E_{0,1}=2.68 \mathrm{eV}\right)$, from which originate the rapid oscillations. Despite their marginal effect on the electron injection rates, the important local influence of correlations on the charge transfer mechanism can be well characterized at the configuration interaction singles level using this new partitioning technique.

\section{CONCLUSIONS}

In this paper, we investigated the ultrafast electron migration process in different dye sensitized solar cell models with special emphasis on the comparison between a timedependent single active electron approach (TDKS) and a timedependent many-electron ansatz (TDCI). To this aim, solar cell models were constructed consisting of the alizarin dye attached to a $\mathrm{TiO}_{2}$ nanocrystallite with two different bonding motifs, i.e., the bridging bidentate and the monodentate. Using linear response TDDFT simulations, the optical spectra of these model systems were first calculated, reproducing the main characteristics of experimental absorption spectra. A static analysis of the electronic properties of the ground and excited states revealed an intrachromophore character for the main band in the absorption spectra. The dominant contribution of this band was found to be a simple HOMO to LUMO transition, which appeared for both model systems at the identical excitation energy of $2.68 \mathrm{eV}$. In essence, the time-independent density based analysis revealed similar charge transfer characteristics for both the model systems and the theoretical approaches. As a result, the manybody character of the TDCI approach was found to play only a minor role for the static properties of this charge transfer system. These static properties are often used to infer electron injection efficiency in dye-sensitized solar cells.

The photoinduced electron dynamics after intrachromophore excitation, followed by charge migration from the dye into the substrate, was studied using explicitly timedependent simulation techniques at various levels of theory. Using absorbing boundary conditions at the edges of the $\mathrm{TiO}_{2}$ cluster to mimic the irreversible electronic injection, we estimated that the electron injection time for the monodentate species is one order of magnitude slower than for the bidentate complex. Despite this fact, the population and dipole moment evolutions showed phenomenologically identical trends for the different model systems. In the context of the methodological comparison, the TDKS approach yielded a quantitatively similar behavior in the dynamical simulation with regard to the reference TDCI method.

The mechanistic pathway of the charge migration process was investigated using a recently developed analysis and imaging toolset based on the time-dependent one-electron densities and electronic flux densities. ${ }^{25}$ To complete the space- and time-resolved analysis of the electron migration process, the electronic yields and electronic fluxes were spatially decomposed using Voronoi tessellation. For the systems selected, an alternating electron flow from the dye to the semiconductor substrate via the bonds of the anchoring hydroxyl groups was found using both levels of theory. The details of the injection mechanism were found to be somewhat different, as TDCI predicted a larger contribution of the dye as a whole due to proper inclusion of correlation in the $N$-electron basis used for the dynamics. This also opened a backward electron transfer channel via a hydrogen bond with the surface during the dye-to-substrate injection step.

From the TDCI wave packet simulations, a novel decomposition technique for the time-dependent electron flux densities was proposed to unravel the correlated nature of the wave function. The visualization and analysis revealed the spatial localization of the electron-electron component at the $\mathrm{TiO}_{2}$ cluster and the predominant distribution of the hole-hole component on the dye. In addition, a slower fluctuation of both contributions was noticed in comparison to the entire electronic flux density. To summarize, the presented work successfully corroborates the suitability of single active electron approach (TDKS) for the description of electron migration processes in DSSCs. The TDKS method represents a low-cost alternative to many-body approaches and thus opens up a wide field for the investigation of more extended systems. Nonetheless, care should be taken in describing the mechanisms, i.e., how the electrons flow at the one-electron level of theory, which cannot describe the dynamical effects of correlation quantitatively.

\section{ACKNOWLEDGMENTS}

The authors acknowledge funding from the Deutsche Forschungsgemeinschaft through Project No. TR1109/2-1 and the Scientific Computing Services Unit of the Zentraleinrichtung für Datenverarbeitung (ZEDAT) at Freie Universität 
Berlin for allocation of computer time. We also thank Hans-Christian Hege for providing the ZIBAmira visualization program and Johannes Dietschreit for stimulating discussions.

${ }^{1}$ R. Miller, Surface Electron Transfer Processes (Wiley-Vch, 1995).

${ }^{2}$ N. A. Anderson and T. Lian, "Ultrafast electron transfer at the moleculesemiconductor nanoparticle interface," Annu. Rev. Phys. Chem. 56, 491-519 (2005).

${ }^{3}$ B. O'Regan and M. Grätzel, "A low-cost, high-efficiency solar cell based on dye-sensitized," Nature 353, 737-740 (1991)

${ }^{4}$ A. Hagfeldt and M. Grätzel, "Light-induced redox reactions in nanocrystalline systems," Chem. Rev. 95, 49-68 (1995).

${ }^{5}$ A. Hagfeldt and M. Grätzel, "Molecular photovoltaics," Acc. Chem. Res. 33, 269-277 (2000).

${ }^{6}$ J. B. Asbury, E. Hao, Y. Wang, H. N. Ghosh, and T. Lian, "Ultrafast electron transfer dynamics from molecular adsorbates to semiconductor nanocrystalline thin films," J. Phys. Chem. B 105, 4545-4557 (2001).

${ }^{7}$ M. Grätzel, "Photoelectrochemical cells," Nature 414, 338-344 (2001).

${ }^{8}$ M. Grätzel, "Dye-sensitized solar cells," J. Photochem. Photobiol., C 4, $145-153$ (2003).

${ }^{9}$ A. Hagfeldt, G. Boschloo, L. Sun, L. Kloo, and H. Pettersson, "Dyesensitized solar cells," Chem. Rev. 110, 6595-6663 (2010).

${ }^{10}$ C. Zimmermann, F. Willig, S. Ramakrishna, B. Burfeindt, B. Pettinger, R. Eichberger, and W. Storck, "Experimental fingerprints of vibrational wavepacket motion during ultrafast heterogeneous electron transfer," J. Phys. Chem. B 105, 9245-9253 (2001).

${ }^{11}$ R. Huber, J.-E. Moser, M. Grätzel, and J. Wachtveitl, "Real-time observation of photoinduced adiabatic electron transfer in strongly coupled dye/semiconductor colloidal systems with a 6 fs time constant," J. Phys. Chem. B 106, 6494-6499 (2002).

${ }^{12}$ J. M. Rehm, G. L. McLendon, Y. Nagasawa, K. Yoshihara, J. Moser, and M. Grätzel, "Femtosecond electron-transfer dynamics at a sensitizing dyesemiconductor $\left(\mathrm{TiO}_{2}\right)$ interface," J. Phys. Chem. 100, 9577-9578 (1996).

${ }^{13}$ R. Huber, J. E. Moser, M. Grätzel, and J. Wachtveitl, "Observation of photoinduced electron transfer in dye/semiconductor colloidal systems with different coupling strengths," Chem. Phys. 285, 39-45 (2002).

${ }^{14}$ J. Schnadt, P. A. Brühwiler, L. Patthey, J. N. O'Shea, S. Södergren, M. Odelius, R. Ahuja, O. Karis, M. Bässler, P. Persson, H. Siegbahn, S. Lunell, and N. Mårtensson, "Experimental evidence for sub-3-fs charge transfer from an aromatic adsorbate to a semiconductor," Nature 418, 620-623 (2002).

${ }^{15}$ S. Ramakrishna and F. Willig, "Pump-probe spectroscopy of ultrafast electron injection from the excited state of an anchored chromophore to a semiconductor surface in UHV: A theoretical model," J. Phys. Chem. B 104, 68-77 (2000)

${ }^{16}$ W. Stier and O. V. Prezhdo, "Nonadiabatic molecular dynamics simulation of light-induced electron transfer from an anchored molecular electron donor to a semiconductor acceptor," J. Phys. Chem. B 106, 8047-8054 (2002).

${ }^{17}$ L. G. Rego and V. S. Batista, "Quantum dynamics simulations of interfacial electron transfer in sensitized $\mathrm{TiO}_{2}$ semiconductors," J. Am. Chem. Soc. 125, 7989-7997 (2003)

${ }^{18} \mathrm{M}$. Thoss, I. Kondov, and H. Wang, "Theoretical study of ultrafast heterogeneous electron transfer reactions at dye-semiconductor interfaces," Chem. Phys. 304, 169-181 (2004).

${ }^{19} \mathrm{~N}$. Martsinovich and A. Troisi, "Theoretical studies of dye-sensitised solar cells: From electronic structure to elementary processes," Energy Environ. Sci. 4, 4473-4495 (2011)

${ }^{20} \mathrm{~W}$. R. Duncan and O. V. Prezhdo, "Theoretical studies of photoinduced electron transfer in dye-sensitized $\mathrm{TiO}_{2}$," Annu. Rev. Phys. Chem. 58, 143-184 (2007).

${ }^{21}$ W. R. Duncan, C. F. Craig, and O. V. Prezhdo, "Time-domain ab initio study of charge relaxation and recombination in dye-sensitized $\mathrm{TiO}_{2}$," J. Am. Chem. Soc. 129, 8528-8543 (2007).

${ }^{22}$ S. G. Abuabara, L. G. Rego, and V. S. Batista, "Influence of thermal fluctuations on interfacial electron transfer in functionalized $\mathrm{TiO}_{2}$ semiconductors," J. Am. Chem. Soc. 127, 18234-18242 (2005).

${ }^{23} \mathrm{~J}$. Li, M. Nilsing, I. Kondov, H. Wang, P. Persson, S. Lunell, and M. Thoss, "Dynamical simulation of photoinduced electron transfer reactions in dyesemiconductor systems with different anchor groups," J. Phys. Chem. C 112, 12326-12333 (2008)

${ }^{24}$ L. Wang, F. Willig, and V. May, "Ultrafast heterogeneous electron transfer reactions: Comparative theoretical studies on time-and frequency-domain data,” J. Chem. Phys. 124, 014712 (2006).
${ }^{25}$ T. Gomez, G. Hermann, X. Zarate, J. F. Pérez-Torres, and J. C. Tremblay, "Imaging the ultrafast photoelectron transfer process in alizarin- $\mathrm{TiO}_{2}$," Molecules 20, 13830-13853 (2015).

${ }^{26} \mathrm{~J}$. Breidbach and L. Cederbaum, "Migration of holes: Formalism, mechanisms, and illustrative applications," J. Chem. Phys. 118, 3983-3996 (2003).

${ }^{27} \mathrm{~J}$. Breidbach and L. Cederbaum, "Migration of holes: Numerical algorithms and implementation," J. Chem. Phys. 126, 034101 (2007).

${ }^{28}$ J. C. Tremblay, S. Klinkusch, T. Klamroth, and P. Saalfrank, "Dissipative many-electron dynamics of ionizing systems," J. Chem. Phys. 134, 044311 (2011).

${ }^{29}$ R. Ramakrishnan and M. Nest, "Charge transfer dynamics from adsorbates to surfaces with single active electron and configuration interaction based approaches," Chem. Phys. 446, 24-29 (2015).

${ }^{30} \mathrm{G}$. Hermann and J. C. Tremblay, "Laser-driven hole trapping in a $\mathrm{Ge} / \mathrm{Si}$ core-shell nanocrystal: An atomistic configuration interaction perspective," J. Phys. Chem. C 119, 25606-25614 (2015).

${ }^{31} \mathrm{H}$. Wang and M. Thoss, "Multilayer formulation of the multiconfiguration time-dependent Hartree theory," J. Chem. Phys. 119, 1289-1299 (2003).

${ }^{32}$ S. Meng and E. Kaxiras, "Real-time, local basis-set implementation of timedependent density functional theory for excited state dynamics simulations," J. Chem. Phys. 129, 054110 (2008).

${ }^{33}$ R. Long and O. V. Prezhdo, "Ab initio nonadiabatic molecular dynamics of the ultrafast electron injection from a pbse quantum dot into the $\mathrm{TiO}_{2}$ surface," J. Am. Chem. Soc. 133, 19240-19249 (2011).

${ }^{34}$ Z. Guo, W. Liang, Y. Zhao, and G. Chen, "Real-time propagation of the reduced one-electron density matrix in atom-centered orbitals: Application to electron injection dynamics in dye-sensitized $\mathrm{TiO}_{2}$ clusters," J. Phys. Chem. C 112, 16655-16662 (2008).

${ }^{35} \mathrm{~J}$. Li, I. Kondov, H. Wang, and M. Thoss, "Theoretical study of photoinduced electron-transfer processes in the dye-semiconductor system alizarin- $\mathrm{TiO}_{2}$," J. Phys. Chem. C 114, 18481-18493 (2010).

${ }^{36}$ R. Huber, S. Spörlein, J. E. Moser, M. Grätzel, and J. Wachtveitl, “The role of surface states in the ultrafast photoinduced electron transfer from sensitizing dye molecules to semiconductor colloids," J. Phys. Chem. B 104, 8995-9003 (2000).

${ }^{37}$ A. Nawrocka and S. Krawczyk, "Electronic excited state of alizarin dye adsorbed on $\mathrm{TiO}_{2}$ nanoparticles: A study by electroabsorption (Stark effect) spectroscopy," J. Phys. Chem. C 112, 10233-10241 (2008).

${ }^{38}$ W. R. Duncan and O. V. Prezhdo, "Electronic structure and spectra of catechol and alizarin in the gas phase and attached to titanium," J. Phys. Chem. B 109, 365-373 (2005).

${ }^{39}$ R. Sánchez-de Armas, J. Oviedo López, M. A. San-Miguel, J. F. Sanz, P. Ordejón, and M. Pruneda, "Real-time TD-DFT simulations in dye sensitized solar cells: The electronic absorption spectrum of alizarin supported on $\mathrm{TiO}_{2}$ nanoclusters," J. Chem. Theory Comput. 6, 2856-2865 (2010).

${ }^{40}$ J. E. Moser and M. Grätzel, "Observation of temperature independent heterogeneous electron transfer reactions in the inverted Marcus region," Chem. Phys. 176, 493-500 (1993).

${ }^{41}$ V. Matylitsky, M. Lenz, and J. Wachtveitl, "Observation of pH-dependent back-electron-transfer dynamics in alizarin/ $/ \mathrm{TiO}_{2}$ adsorbates: Importance of trap states," J. Phys. Chem. B 110, 8372-8379 (2006).

${ }^{42}$ L. Dworak, V. V. Matylitsky, and J. Wachtveitl, "Ultrafast photoinduced processes in alizarin-sensitized metal oxide mesoporous films," ChemPhysChem 10, 384-391 (2009).

${ }^{43}$ W. R. Duncan, W. M. Stier, and O. V. Prezhdo, "Ab Initio nonadiabatic molecular dynamics of the ultrafast electron injection across the alizarin$\mathrm{TiO}_{2}$ interface," J. Am. Chem. Soc. 127, 7941-7951 (2005).

${ }^{44}$ S. Kaniyankandy, S. Verma, J. A. Mondal, D. K. Palit, and H. N. Ghosh, "Evidence of multiple electron injection and slow back electron transfer in alizarin-sensitized ultrasmall $\mathrm{TiO}_{2}$ particles," J. Phys. Chem. C 113, 3593-3599 (2009).

${ }^{45}$ W. Stier, W. R. Duncan, and O. V. Prezhdo, "Thermally assisted sub-10 fs electron transfer in dye-sensitized nanocrystalline $\mathrm{TiO}_{2}$ solar cells," Adv. Mater. 16, 240-244 (2004).

${ }^{46}$ J. K. Burdett, T. Hughbanks, G. J. Miller, J. W. R., Jr., and J. V. Smith, "Structural-electronic relationships in inorganic solids: Powder neutron diffraction studies of the rutile and anatase polymorphs of titanium dioxide at 15 and $295 \mathrm{~K}$," J. Am. Chem. Soc. 109, 3639-3646 (1987).

${ }^{47}$ I. Kondov, H. Wang, and M. Thoss, "Computational study of titanium (Iv) complexes with organic chromophores," Int. J. Quantum Chem. 106, 1291-1303 (2006)

${ }^{48}$ A. D. Becke, "Density-functional exchange-energy approximation with correct asymptotic behavior," Phys. Rev. A 38, 3098-3100 (1988).

${ }^{49}$ A. Schäfer, H. Horn, and R. Ahlrichs, "Fully optimized contracted Gaussian basis sets for atoms Li to Kr," J. Chem. Phys. 97, 2571-2577 (1992). 
${ }^{50} \mathrm{~F}$. Weigend and R. Ahlrichs, "Balanced basis sets of split valence, triple zeta valence and quadruple zeta valence quality for $\mathrm{H}$ to $\mathrm{Rn}$ : Design and assessment of accuracy," Phys. Chem. Chem. Phys. 7, 3297-3305 (2005).

${ }^{51} \mathrm{E}$. Gross and W. Kohn, "Time-dependent density functional theory," Adv. Quantum Chem. 21, 255-291 (1990).

${ }^{52} \mathrm{~F}$. De Angelis, A. Tilocca, and A. Selloni, "Time-dependent DFT study of $\left[\mathrm{Fe}(\mathrm{CN})_{6}\right]^{4-}$ sensitization of $\mathrm{TiO}_{2}$ nanoparticles," J. Am. Chem. Soc. 126, 15024-15025 (2004).

${ }^{53}$ P. Persson and M. J. Lundqvist, "Calculated structural and electronic interactions of the ruthenium dye N3 with a titanium dioxide nanocrystal," J. Phys. Chem. B 109, 11918-11924 (2005).

${ }^{54}$ P. Persson, M. J. Lundqvist, R. Ernstorfer, W. Goddard, and F. Willig, "Quantum chemical calculations of the influence of anchor-cum-spacer groups on femtosecond electron transfer times in dye-sensitized semiconductor nanocrystals," J. Chem. Theory Comput. 2, 441-451 (2006).

${ }^{55}$ F. De Angelis, S. Fantacci, A. Selloni, M. K. Nazeeruddin, and M. Grätzel, "Time-dependent density functional theory investigations on the excited states of $\mathrm{Ru}(\mathrm{II})$-dye-sensitized $\mathrm{TiO}_{2}$ nanoparticles: The role of sensitizer protonation," J. Am. Chem. Soc. 129, 14156-14157 (2007).

${ }^{56}$ D. Rocca, R. Gebauer, F. De Angelis, M. K. Nazeeruddin, and S. Baroni, "Time-dependent density functional theory study of squaraine dyesensitized solar cells," Chem. Phys. Lett. 475, 49-53 (2009).

57"'TURBOMOLE V6.5, a development of University of Karlsruhe and Forschungszentrum Karlsruhe GmbH, 1989-2007, TURBOMOLE GmbH, since 2007," 2013, available via http://www.turbomole.com.

${ }^{58}$ G. Hermann, V. Pohl, J. C. Tremblay, B. Paulus, H.-C. Hege, and A. Schild, "ORBKIT: A modular python toolbox for cross-platform postprocessing of quantum chemical wavefunction data," J. Comput. Chem. 37, 1511-1520 (2016).

${ }^{59}$ E. Schrödinger, "Quantisierung als Eigenwertproblem," Ann. Phys. 386, 109-139 (1926).

${ }^{60}$ J. C. Tremblay and T. Carrington, Jr., "Using preconditioned adaptive step size Runge-Kutta methods for solving the time-dependent Schrödinger equation," J. Chem. Phys. 121, 11535-11541 (2004).

${ }^{61}$ J. C. Tremblay, T. Klamroth, and P. Saalfrank, "Time-dependent configuration-interaction calculations of laser-driven dynamics in presence of dissipation," J. Chem. Phys. 129, 084302 (2008).

${ }^{62}$ J. B. Foresman, M. Head-Gordon, J. A. Pople, and M. J. Frisch, "Toward a systematic molecular orbital theory for excited states," J. Phys. Chem. 96, 135-149 (1992).

${ }^{63}$ J. A. Sonk and H. B. Schlegel, "TD-CI simulation of the strong-field ionization of polyenes,” J. Phys. Chem. A 116, 7161-7168 (2012).

${ }^{64} \mathrm{~S}$. Klinkusch and J. C. Tremblay, "Resolution-of-identity stochastic timedependent configuration interaction for dissipative electron dynamics in strong fields," J. Chem. Phys. 144, 184108 (2016).

${ }^{65}$ P. Elliott, S. Goldson, C. Canahui, and N. T. Maitra, "Perspectives on doubleexcitations in TDDFT," Chem. Phys. 391, 110-119 (2011).
${ }^{66}$ M. Pastore, E. Mosconi, F. D. Angelis, and M. Grätzel, “A computational investigation of organic dyes for dye-sensitized solar cells: Benchmark, strategies, and open issues," J. Phys. Chem. C 114, 7205-7212 (2010).

${ }^{67} \mathrm{~F}$. Jensen, Introduction to Computational Chemistry (John Wiley \& Sons, 2013).

${ }^{68} \mathrm{~L}$. Nafie, "Electron transition current density in molecules. 1. Non-BornOppenheimer theory of vibronic and vibrational transitions," J. Phys. Chem. A 101, 7826-7833 (1997).

${ }^{69}$ C. Fonseca Guerra, J.-W. Handgraaf, E. J. Baerends, and F. M. Bickelhaupt, "Voronoi deformation density (VDD) charges: Assessment of the Mulliken, Bader, Hirshfeld, Weinhold, and VDD methods for charge analysis," J. Comput. Chem. 25, 189-210 (2004).

${ }^{70}$ T. B. Freedman, X. Gao, M.-L. Shih, and L. A. Nafie, "Electron transition current density in molecules. 2. Ab initio calculations for electronic transitions in ethylene and formaldehyde," J. Phys. Chem. A 102, 3352-3357 (1998).

${ }^{71}$ G. Hermann, B. Paulus, J. Pérez-Torres, and V. Pohl, "Electronic and nuclear flux densities in the $\mathrm{H}_{2}$ molecule," Phys. Rev. A 89, 052504 (2014).

${ }^{72}$ I. Barth, H.-C. Hege, H. Ikeda, A. Kenfack, M. Koppitz, J. Manz, F. Marquardt, and G. K. Paramonov, "Concerted quantum effects of electronic and nuclear fluxes in molecules," Chem. Phys. Lett. 481, 118-123 (2009).

${ }^{73}$ M. Berg, B. Paulus, and T. Bredtmann, "Electronic quantum fluxes in vibrating symmetric and polar single, double and triple bonds," Mol. Phys. 114, 1356-1364 (2015).

${ }^{74}$ G. Hermann, C. Liu, J. Manz, B. Paulus, J. F. Pérez-Torres, V. Pohl, and J. C. Tremblay, "Multidirectional angular electronic flux during adiabatic attosecond charge migration in excited benzene," J. Phys. Chem. A 120, 5360-5369 (2016).

${ }^{75}$ D. Stalling, M. Westerhoff, and H.-C. Hege, "38 - amira: A highly interactive system for visual data analysis," in Visualization Handbook, edited by C. D. Hansen and C. R. Johnson (Elsevier, Amsterdam, 2005), Chap. 8, pp. 749-767.

${ }^{76}$ P. Redfern, P. Zapol, L. Curtiss, T. Rajh, and M. Thurnauer, "Computational studies of catechol and water interactions with titanium oxide nanoparticles," J. Phys. Chem. B 107, 11419-11427 (2003).

${ }^{77}$ S. Grimme, J. Antony, S. Ehrlich, and H. Krieg, "A consistent and accurate $a b$ initio parametrization of density functional dispersion correction (DFTD) for the 94 elements H-Pu," J. Chem. Phys. 132, 154104 (2010).

${ }^{78}$ T. Le Bahers, C. Adamo, and I. Ciofini, "A qualitative index of spatial extent in charge-transfer excitations," J. Chem. Theory Comput. 7, 2498-2506 (2011).

${ }^{79}$ T. Le Bahers, T. Pauporté, P. P. Lainé, F. Labat, C. Adamo, and I. Ciofini, "Modeling dye-sensitized solar cells: From theory to experiment," J. Phys. Chem. Lett. 4, 1044-1050 (2013).

${ }^{80}$ M. Zöckler, D. Stalling, and H.-C. Hege, "Interactive visualization of 3D-vector fields using illuminated stream lines," in Proceedings of Visualization'96 (IEEE, 1996), pp. 107-113. 